\title{
DIRAC STRUCTURES FOR GENERALIZED LIE BIALGEBROIDS
}

\author{
J. M. NUNES DA COSTA AND J. CLEMENTE-GALLARDO
}

\begin{abstract}
We introduce the notion of Dirac structure for a generalized Courant algebroid. We show that the double of a generalized Lie bialgebroid is a generalized Courant algebroid. We present some examples and we obtain, as a particular case of our definition, the notion of $\mathcal{E}^{1}(M)$-Dirac structure introduced by Wade.
\end{abstract}

KEYWORDS: Dirac structures, generalized Courant algebroids, generalized Lie bialgebroids, Jacobi manifolds.

AMS SubJeCt ClassificAtion (2000): 17B62, 53D10, 53D17.

\section{Introduction}

Dirac structures on manifolds were introduced by Courant and Weinstein [3] and studied in detail in [2]. Dirac structures include closed 2-forms, Poisson structures and foliations. Dorfman [4] developed an algebraic treatment of these structures and used them for the study of completely integrable systems, in the context of the calculus of variations.

The notion of Dirac structure on a manifold $M$, investigated by Courant in [2], is defined using a subbundle $L$ of $T M \oplus T^{*} M$, which is maximally isotropic under the natural symmetric pairing on $T M \oplus T^{*} M$, and a bracket on the space of sections of $T M \oplus T^{*} M$, called the Courant bracket. The existence of a Dirac structure corresponds to the closedness of that bracket on the space $\Gamma(L)$ of sections of $L$.

In order to understand the meaning of this bracket, which is not a Lie bracket on $\Gamma\left(T M \oplus T^{*} M\right)$, Liu et al [16] introduced the notion of Courant algebroid on a vector bundle whose definition includes a skew-symmetric bracket on the space of sections of that bundle. The first example of Courant algebroid is the Whitney sum bundle $A \oplus A^{*}$, where the pair $\left(A, A^{*}\right)$ is a Lie bialgebroid [18]. The Courant algebroid $A \oplus A^{*}$ is called the double of the Lie bialgebroid $\left(A, A^{*}\right)$

For the case of the Lie bialgebroid $\left(T M, T^{*} M\right)$, where $T M$ is the Lie algebroid whose space of sections is endowed with the usual Lie bracket of vector fields and $T^{*} M$ is the null Lie algebroid, the bracket on the Courant algebroid $T M \oplus T^{*} M$ is the one introduced in [2], i.e. the Courant bracket. 
It is well known that there exists a close relation between Lie bialgebroids and Poisson structures on manifolds. However, if we pass from the Poisson to the Jacobi setting, we have to replace Lie bialgebroids by generalized Lie bialgebroids [6] or Jacobi bialgebroids [5]. In fact, in opposite to the Poisson case, the pair $\left(T M, T^{*} M\right)$ is not, in general, a Lie bialgebroid if $M$ is a Jacobi manifold. Canonically associated with a Jacobi manifold $M$, there is a generalized Lie bialgebroid structure on $\left(T M \times \mathbb{R}, T^{*} M \times \mathbb{R}\right)$. In [23], Wade considered the Whitney sum bundle $\mathcal{E}^{1}(M)=(T M \times \mathbb{R}) \oplus\left(T^{*} M \times \mathbb{R}\right)$ and introduced the notion of $\mathcal{E}^{1}(M)$-Dirac structure, extending the Courant bracket to the space of sections of $\mathcal{E}^{1}(M)$. But this extended bracket in not a Courant bracket.

The motivation for this paper was a tentative to understand the exact meaning of the extended bracket introduced by Wade, and to see how it is related with the Courant algebroid structure. For that, we introduce the notion of a generalized Courant algebroid, and we show that this includes the double of a generalized Lie bialgebroid. We then conclude that the bracket introduced by Wade is the bracket of a generalized Courant algebroid, defined on $\mathcal{E}^{1}(M)$, for the case where $T^{*} M \times \mathbb{R}$ is endowed with the null Lie algebroid structure.

The paper is organized as follows. In section 2 we recall some definitions and results concerning generalized Lie bialgebroids and Jacobi manifolds. In section 3 we present the definitions of generalized Courant algebroid and Dirac structure for a generalized Courant algebroid. We prove that the double of a generalized Lie bialgebroid is a generalized Courant algebroid and we recover, as an example, the $\mathcal{E}^{1}(M)$-Dirac structure introduced by Wade in [23]. Sections 4 and 5 are devoted to the presentation of some examples of Dirac structures for generalized Lie bialgebroids $\left((A, \phi),\left(A^{*}, W\right)\right)$. In section 4 we study the graph of a bivector field on $A$, i.e. a section of $\bigwedge^{2} A$, and we investigate the case of a Jacobi structure $(\Lambda, E) \in \Gamma\left(\bigwedge^{2}(T M \times \mathbb{R})\right)$ on $M$. In section 5 we consider a subbundle $D \subset A$ of $A$ and we establish the conditions that ensure the existence of a Dirac structure on the vector bundle $D \oplus D^{\perp}$, where $D^{\perp}$ is the conormal bundle of $D$. Using the notion of characteristic pair [15] of a subbundle $A$, we obtain another example of a Dirac structure. Finally, in section 6 we consider the Dirac structure for a triangular generalized Lie bialgebroid. 


\section{Generalized Lie bialgebroids and Jacobi structures}

A Lie algebroid $(A,[.,],. \rho)$ over a manifold $M$ is a vector bundle $A$ over $M$ together with a bundle map $\rho: A \rightarrow T M$, called the anchor and a Lie algebra structure $[.,$.$] on the space \Gamma(A)$ of the global cross sections such that

i) the induced map $\rho: \Gamma(A) \rightarrow \mathfrak{X}(M)$ is a Lie algebra homomorphism;

ii) for any $f \in C^{\infty}(M, \mathbb{R})$ and $X, Y \in \Gamma(A)$, then

$$
[X, f Y]=f[X, Y]+(\rho(X) f) Y .
$$

Example 2.1. If $M$ is a differentiable manifold, then the triple $\left(T M,[.,],. I d_{T M}\right)$ is a Lie algebroid over $M$, where [.,.] is the usual Lie bracket of vector fields.

Notation: Throughout this paper, we will use $\delta$ to denote the usual differential of de Rham.

Example 2.2. Let $(M, \Lambda)$ be a Poisson manifold and $\Lambda^{\#}: T^{*} M \rightarrow T M$ the vector bundle morphism associated with the Poisson tensor $\Lambda$ given, for any sections $\alpha, \beta$ of $T^{*} M$, by

$$
\left\langle\beta, \Lambda^{\#}(\alpha)\right\rangle=\Lambda(\alpha, \beta) .
$$

Then the triple $\left(T^{*} M,[., \cdot]_{\Lambda}, \Lambda^{\#}\right)$ is a Lie algebroid over $M$, where $[., .]_{\Lambda}$ is the Lie bracket of 1-forms given by

$$
[\alpha, \beta]_{\Lambda}=\mathcal{L}_{\Lambda^{\#}(\alpha)} \beta-\mathcal{L}_{\Lambda^{\#}(\beta)} \alpha-\delta(\Lambda(\alpha, \beta)) .
$$

We recall that a Jacobi structure on a manifold $M$ is a pair $(\Lambda, E)$, where $\Lambda$ is a bivector and $E$ is a vector field such that $[\Lambda, \Lambda]=-2 E \wedge \Lambda$ and $[E, \Lambda]=0,[14]$.

Example 2.3. Let $(M, \Lambda, E)$ be a Jacobi manifold. We denote by $(\Lambda, E)^{\#}$ : $T^{*} M \times \mathbb{R} \rightarrow T M \times \mathbb{R}$ the vector bundle morphism given by

$$
(\Lambda, E)^{\#}(\alpha, f)=\left(\Lambda^{\#}(\alpha)+f E,-\langle\alpha, E\rangle\right),
$$

for any section $\alpha$ of $T^{*} M$ and $f \in C^{\infty}(M, \mathbb{R})$. In opposition to the case of a Poisson manifold, in general one cannot define a Lie algebroid structure on the cotangent bundle of a Jacobi manifold. However, if $(M, \Lambda, E)$ is a Jacobi manifold, then $\left(T^{*} M \times \mathbb{R},[., .]_{(\Lambda, E)}, \pi \circ(\Lambda, E)^{\#}\right)$ is a Lie algebroid over $M$ 
[9], where $\pi: T M \times \mathbb{R} \rightarrow T M$ is the projection over the first factor and $[., .]_{(\Lambda, E)}$ is the bracket given by

$$
[(\alpha, f),(\beta, g)]_{(\Lambda, E)}:=(\gamma, r),
$$

with

$$
\begin{gathered}
\gamma:=\mathcal{L}_{\Lambda^{\#}(\alpha)} \beta-\mathcal{L}_{\Lambda^{\#}(\beta)} \alpha-\delta(\Lambda(\alpha, \beta))+f \mathcal{L}_{E} \beta-g \mathcal{L}_{E} \alpha-i_{E}(\alpha \wedge \beta), \\
r:=-\Lambda(\alpha, \beta)+\Lambda(\alpha, \delta g)-\Lambda(\beta, \delta f)+\langle f \delta g-g \delta f, E\rangle .
\end{gathered}
$$

It is well known that with each Lie algebroid $(A,[.,],. \rho)$ a differential $d$ on the graded space of sections of $\bigwedge A^{*}=\oplus_{k \in \mathbb{Z}} \bigwedge^{k} A^{*}$ is associated, where $A^{*}$ is the dual vector bundle of $A$. More precisely, $d$ is a derivation of degree 1 and of square 0 of the associative graded commutative algebra $\left(\Gamma\left(\bigwedge A^{*}\right), \wedge\right)$. Also the Lie bracket on $\Gamma(A)$ can be extended to the algebra of sections of $\bigwedge A, \Gamma(\bigwedge A)=\oplus_{k \in \mathbb{Z}} \Gamma\left(\bigwedge^{k} A\right)$. The result is a graded Lie bracket [., .] which is called the Schouten bracket of the Lie algebroid. ${ }^{1}$ For more details, see [17] and [10].

Let $(A,[.,],. \rho)$ be a Lie algebroid over $M$ and $\phi \in \Gamma\left(A^{*}\right)$ a 1-cocycle for the Lie algebroid cohomology complex with trivial coefficients (see [17] and [6] ), i.e. for all $X, Y \in \Gamma(A)$,

$$
\langle\phi,[X, Y]\rangle=\rho(X)(\langle\phi, Y\rangle)-\rho(Y)(\langle\phi, X\rangle) .
$$

Using the 1-cocycle $\phi$, we can define a new representation $\rho^{\phi}$ of the Lie algebra $(\Gamma(A),[.,]$.$) on C^{\infty}(M, \mathbb{R})$, by setting

$\rho^{\phi}: \Gamma(A) \times C^{\infty}(M, \mathbb{R}) \rightarrow C^{\infty}(M, \mathbb{R}),(X, f) \mapsto \rho^{\phi}(X, f)=\rho(X) f+\langle\phi, X\rangle f$.

Therefore, we obtain a new cohomology complex, whose differential cohomology operator is given by

$$
d^{\phi}: \Gamma\left(\bigwedge^{k} A^{*}\right) \rightarrow \Gamma\left(\bigwedge^{k+1} A^{*}\right), \quad \beta \mapsto d^{\phi}(\beta)=d \beta+\phi \wedge \beta .
$$

Also, for any $X \in \Gamma(A)$, the Lie derivative operator with respect to $X$ is given by

$$
\mathcal{L}_{X}^{\phi}: \Gamma\left(\bigwedge^{k} A^{*}\right) \rightarrow \Gamma\left(\bigwedge^{k} A^{*}\right), \quad \beta \mapsto \mathcal{L}_{X}^{\phi}(\beta)=\mathcal{L}_{X} \beta+\langle\phi, X\rangle \beta .
$$

\footnotetext{
${ }^{1}$ Some differences in signs with [6] and [14] come from different conventions for the Schouten bracket.
} 
It is also possible to consider a $\phi$-Schouten bracket on the graded algebra $\Gamma(\bigwedge A)$, denoted by $[., .]^{\phi}$, which is defined as follows:

$$
\begin{aligned}
& {[., .]^{\phi}: \Gamma\left(\bigwedge^{p} A\right) \times \Gamma\left(\bigwedge^{q} A\right) \rightarrow \Gamma\left(\bigwedge^{p+q-1} A\right)} \\
& \quad(P, Q) \mapsto[P, Q]^{\phi}=[P, Q]+(p-1) P \wedge\left(i_{\phi} Q\right)+(-1)^{p}(q-1)\left(i_{\phi} P\right) \wedge Q,
\end{aligned}
$$

where $i_{\phi} Q$ can be interpreted as the usual contraction of a multivector field by a 1 -form. We observe that when $p=q=1,[P, Q]^{\phi}=[P, Q]$. That is, the brackets $[., .]^{\phi}$ and $[.,$.$] coincide on \Gamma(A)$.

We can develop a differential calculus using $\rho^{\phi}, d^{\phi}, \mathcal{L}^{\phi}$ and [.,.] $]^{\phi}$. The formulae obtained are similar, but adapted, to the case of a Lie algebroid (see [6] and [5]).

Suppose that the vector bundle $(A,[.,],. \rho)$ and its dual vector bundle $\left(A^{*},[.,]_{*}, \rho_{*}\right)$ are both Lie algebroids over a manifold $M$. Let $d$ (resp. $\left.d_{*}\right)$ denote the differential of $A$ (resp. $\left.A^{*}\right)$. Let $\phi \in \Gamma\left(A^{*}\right)$ (resp. $\left.W \in \Gamma(A)\right)$ be a 1-cocycle in the Lie algebroid cohomology complex of $(A,[.,],. \rho)$ (resp. $\left.\left(A^{*},[., .]_{*}, \rho_{*}\right)\right)$.

Definition 2.4. ([6]) The pair $\left((A, \phi),\left(A^{*}, W\right)\right)$ is a generalized Lie bialgebroid if for all $X, Y \in \Gamma(A)$ and $P \in \Gamma\left(\bigwedge^{p} A\right)$, the following conditions hold:

$$
\begin{gathered}
d_{*}^{W}[X, Y]=\left[d_{*}^{W} X, Y\right]^{\phi}+\left[X, d_{*}^{W} Y\right]^{\phi} ; \\
\mathcal{L}_{* \phi}^{W} P+\mathcal{L}_{W}^{\phi} P=0 .
\end{gathered}
$$

Under the name of Jacobi bialgebroid, this notion was presented in [5], with the following definition:

Definition 2.5. ([5]) The pair $\left((A, \phi),\left(A^{*}, W\right)\right)$ is a Jacobi bialgebroid if for all $P \in \Gamma\left(\bigwedge^{p} A\right)$ and $Q \in \Gamma(\bigwedge A)$,

$$
d_{*}^{W}[P, Q]^{\phi}=\left[d_{*}^{W} P, Q\right]^{\phi}+(-1)^{p+1}\left[P, d_{*}^{W} Q\right]^{\phi} .
$$

The equivalence of Definitions 2.4 and 2.5 was proved in [5].

When $\phi=0$ and $W=0$, we recover the notion of Lie bialgebroid: Definition 2.4 generalizes the original definition introduced in [18] by K. Mackenzie 
and $\mathrm{P} . \mathrm{Xu}$, while Definition 2.5 generalizes the equivalent one given by $\mathrm{Y}$. Kosmann-Schwarzbach [10].

The important property of duality of a Lie bialgebroid is also verified in the case of a generalized Lie bialgebroid: if $\left((A, \phi),\left(A^{*}, W\right)\right)$ is a generalized Lie bialgebroid, so is $\left(\left(A^{*}, W\right),(A, \phi)\right)$ (see [6] and [5]). As a consequence, both Definitions 2.4 and 2.5 can be given using the dual versions of (9)-(10) and (11), respectively.

Example 2.6. Let $(M, \Lambda, E)$ be a Jacobi manifold. Consider the associated Lie algebroid $\left(T^{*} M \times \mathbb{R},[.,]_{(\Lambda, E)}, \pi \circ(\Lambda, E)^{\#}\right)$ over $M$. Its differential $d_{*}$ is given for all $(P, Q) \in \Gamma\left(\bigwedge^{k}(T M)\right) \oplus \Gamma\left(\bigwedge^{k-1}(T M)\right)$, by ([13])

$$
d_{*}(P, Q)=([\Lambda, P]+k E \wedge P+\Lambda \wedge Q,-[\Lambda, Q]+(1-k) E \wedge Q+[E, P]) .
$$

On the other hand, if $M$ is a differentiable manifold, then the triple $(T M \times$ $\mathbb{R},[.,],. \pi)$ is a Lie algebroid over $M$, where $\pi$ is the projection over the first factor and $[.,$.$] is given by$

$[(X, f),(Y, g)]=([X, Y], X(g)-Y(f)),(X, f),(Y, g) \in \mathfrak{X}(M) \times C^{\infty}(M, \mathbb{R})$

The associated differential is $d=(\delta,-\delta), \delta$ being the de Rham differential.

In [6] it was proved that $\phi=(0,1)$ (resp. $W=(-E, 0)$ ) is a 1-cocycle of $T M \times \mathbb{R}\left(\right.$ resp. $\left.T^{*} M \times \mathbb{R}\right)$ and the pair $\left((T M \times \mathbb{R}, \phi),\left(T^{*} M \times \mathbb{R}, W\right)\right)$ is a Jacobi bialgebroid.

Another interesting example of generalized Lie bialgebroid is the one provided by strict Jacobi-Nijenhuis manifolds (see [21] and [8]).

\section{Generalized Courant algebroids}

In this section we introduce the notion of generalized Courant algebroid and we show that the double of a generalized Lie bialgebroid is a generalized Courant algebroid.

Definition 3.1. A generalized Courant algebroid is a pair $(A, \theta)$, where $A$ is a vector bundle $A \rightarrow M$ equipped with a nondegenerate symmetric bilinear form (.,.) on the bundle, a skew-symmetric bracket [.,.] on $\Gamma(A)$ and a bundle map $\rho: A \rightarrow T M$, and $\theta \in \Gamma\left(A^{*}\right)$ is such that, for any $X, Y \in \Gamma(A)$, $\langle\theta,[X, Y]\rangle=\rho(X)\langle\theta, Y\rangle-\rho(Y)\langle\theta, X\rangle$, verifying the following properties: 
(1) for any $X_{1}, X_{2}, X_{3} \in \Gamma(A)$,

$$
\left[\left[X_{1}, X_{2}\right], X_{3}\right]+\text { c.p. }=\mathcal{D}^{\theta} T\left(X_{1}, X_{2}, X_{3}\right) ;
$$

(2) for any $X_{1}, X_{2} \in \Gamma(A)$,

$$
\rho\left(\left[X_{1}, X_{2}\right]\right)=\left[\rho\left(X_{1}\right), \rho\left(X_{2}\right)\right]
$$

(3) for any $X_{1}, X_{2} \in \Gamma(A)$ and $f \in C^{\infty}(M, \mathbb{R})$,

$$
\left[X_{1}, f X_{2}\right]=f\left[X_{1}, X_{2}\right]+\left(\rho\left(X_{1}\right) f\right) X_{2}-\left(X_{1}, X_{2}\right) \mathcal{D} f
$$

(4) for any $f, g \in C^{\infty}(M, \mathbb{R})$,

$$
\left(\mathcal{D}^{\theta} f, \mathcal{D}^{\theta} g\right)
$$

(5) for any $Y, X_{1}, X_{2} \in \Gamma(A)$,

$$
\begin{aligned}
\rho(Y)\left(X_{1}, X_{2}\right)+\langle\theta, Y\rangle\left(X_{1}, X_{2}\right)= & \left(\left[Y, X_{1}\right]+\mathcal{D}^{\theta}\left(Y, X_{1}\right), X_{2}\right) \\
& +\left(X_{1},\left[Y, X_{2}\right]+\mathcal{D}^{\theta}\left(Y, X_{2}\right)\right),
\end{aligned}
$$

where $T\left(X_{1}, X_{2}, X_{3}\right)$ is the function defined by

$$
T\left(X_{1}, X_{2}, X_{3}\right)=\frac{1}{3}\left(\left[X_{1}, X_{2}\right], X_{3}\right)+c . p .
$$

and $\mathcal{D}, \mathcal{D}^{\theta}: C^{\infty}(M, \mathbb{R}) \rightarrow \Gamma(A)$ are given, for any $X \in \Gamma(A)$, respectively by

$$
\left(\mathcal{D}^{\theta} f, X\right)=\frac{1}{2}(\rho(X) f+\langle\theta, X\rangle f) \quad \text { and } \quad(\mathcal{D} f, X)=\frac{1}{2} \rho(X) f .
$$

When $\theta=0$, we recover the definition of Courant algebroid, introduced in [16], or the equivalent version presented in [22].

A subbundle $L \subset A$ of the generalized Courant algebroid $(A, \theta)$ is said to be integrable if $\Gamma(L)$ is closed under the bracket [.,.].

Definition 3.2. A Dirac structure for the generalized Courant algebroid $(A, \theta)$ is an integrable subbundle $L$ of $A$ which is maximally isotropic with respect to the symmetric bilinear form (.,.).

An immediate consequence of the previous definition is the following.

Proposition 3.3. If $L$ is a Dirac structure for the generalized Courant algebroid $(A, \theta)$ and $\theta \in \Gamma\left(L^{*}\right)$, then $\left(L,\left.\rho\right|_{L},\left.[.,]\right|_{L}.\right)$ is a Lie algebroid and $\theta$ is a 1-cocycle for the Lie algebroid cohomology complex with trivial coefficients. 
Suppose now that the vector bundle $A \rightarrow M$ and its dual $A^{*} \rightarrow M$ are both equipped with Lie algebroid structures $([.,], a$.$) and \left([., .]_{*}, a_{*}\right)$, respectively. Let $d\left(\right.$ resp. $\left.d_{*}\right)$ denote the differential of $A$ (resp. $\left.A^{*}\right)$ and let $\phi \in \Gamma\left(A^{*}\right)$ (resp. $W \in \Gamma(A))$ be a 1-cocycle in the Lie algebroid cohomology complex of $(A,[.,], a$.$\left.) (resp. \left(A^{*},[., .]_{*}, a_{*}\right)\right)$. Moreover, let $d^{\phi}, \mathcal{L}^{\phi}$ and $[., .]^{\phi}$ (resp. $d_{*}^{W}, \mathcal{L}_{*}^{W}$ and $\left.[.,]_{*}^{W}\right)$ be the differential, the Lie derivative and the bracket modified by the 1-cocycle $\phi$ of $A$ (resp. $W$ of $A^{*}$ ) given by (6), (7) and (8), respectively.

On the Whitney sum bundle $A \oplus A^{*}$ we can define two nondegenerate bilinear forms, one symmetric, denoted by $(., .)_{+}$, and the other skew-symmetric, denoted by $(., .)_{-}$, by setting, for any $X_{1}+\alpha_{1}, X_{2}+\alpha_{2} \in A \oplus A^{*}$,

$$
\left(X_{1}+\alpha_{1}, X_{2}+\alpha_{2}\right)_{+}=\frac{1}{2}\left(\left\langle\alpha_{1}, X_{2}\right\rangle+\left\langle\alpha_{2}, X_{1}\right\rangle\right)
$$

and

$$
\left(X_{1}+\alpha_{1}, X_{2}+\alpha_{2}\right)_{-}=\frac{1}{2}\left(\left\langle\alpha_{1}, X_{2}\right\rangle-\left\langle\alpha_{2}, X_{1}\right\rangle\right),
$$

respectively.

On the space $\Gamma\left(A \oplus A^{*}\right)$ of the global cross sections of $A \oplus A^{*}$, which is identified with $\Gamma(A) \oplus \Gamma\left(A^{*}\right)$, we introduce the following bracket:

$$
\begin{aligned}
\llbracket X_{1}+\alpha_{1}, X_{2}+\alpha_{2} \rrbracket= & \left(\left[X_{1}, X_{2}\right]^{\phi}+\mathcal{L}_{* \alpha_{1}}^{W} X_{2}-\mathcal{L}_{* \alpha_{2}}^{W} X_{1}-d_{*}^{W}\left(e_{1}, e_{2}\right)_{-}\right) \\
& +\left(\left[\alpha_{1}, \alpha_{2}\right]_{*}^{W}+\mathcal{L}_{X_{1}}^{\phi} \alpha_{2}-\mathcal{L}_{X_{2}}^{\phi} \alpha_{1}+d^{\phi}\left(e_{1}, e_{2}\right)_{-}\right)
\end{aligned}
$$

where $e_{1}=X_{1}+\alpha_{1}$ and $e_{2}=X_{2}+\alpha_{2}$.

Using the anchor maps $a$ and $a_{*}$, we define a vector bundle map $\rho: A \oplus$ $A^{*} \rightarrow T M$ which is given, for any section $X+\alpha$ of $A \oplus A^{*}$, by

$$
\rho(X+\alpha)=a(X)+a_{*}(\alpha) .
$$

We have now all the ingredients for establishing the main result of this section.

Theorem 3.4. If $\left((A, \phi),\left(A^{*}, W\right)\right)$ is a generalized Lie bialgebroid over $M$, then the pair $\left(A \oplus A^{*}, \theta\right)$, with $\theta=\phi+W$ is a generalized Courant algebroid with the Lie bracket $\llbracket . . . \rrbracket$ on $\Gamma\left(A \oplus A^{*}\right)$ given by (23), the symmetric bilinear form given by (21), the vector bundle map $\rho$ given by (24)and the operators $\mathcal{D}, \mathcal{D}^{\theta}$ given respectively by $\mathcal{D}=\left(d+d_{*}\right)_{\mid C^{\infty}(M, \mathbb{R})}$ and $\mathcal{D}^{\theta}=\left(d^{\phi}+d_{*}^{W}\right)_{\mid C^{\infty}(M, \mathbb{R})}$. 
Before proving this theorem, we need some technical lemmas.

Lemma 3.5. Let $\left((A, \phi),\left(A^{*}, W\right)\right)$ be a generalized Lie bialgebroid over $M$. Then,

i) $\langle\phi, W\rangle=0, \quad a(W)+a_{*}(\phi)=0$ and, for any $X \in \Gamma(A), \alpha \in \Gamma\left(A^{*}\right)$,

$$
\mathcal{L}_{* \phi} X+[W, X]=0, \quad \mathcal{L}_{W} \alpha+[\phi, \alpha]_{*}=0 ;
$$

ii) for any $X \in \Gamma(A), \alpha \in \Gamma\left(A^{*}\right)$ and $f \in C^{\infty}(M, \mathbb{R})$,

$$
\left[d_{*}^{W} f, X\right]^{\phi}+\mathcal{L}_{* d^{\phi} f}^{W} X=0, \quad\left[d^{\phi} f, \alpha\right]_{*}^{W}+\mathcal{L}_{d_{*}^{W} f}^{\phi} \alpha=0 ;
$$

iii) for any $f \in C^{\infty}(M, \mathbb{R})$,

$$
\left(a \circ d_{*}^{W}+a_{*} \circ d^{\phi}\right) f=0 ;
$$

iv) for any $f, g \in C^{\infty}(M, \mathbb{R})$,

$$
\left\langle d^{\phi} f, d_{*}^{W} g\right\rangle+\left\langle d^{\phi} g, d_{*}^{W} f\right\rangle=0 .
$$

Proof: $\quad$ i) The first three equalities follow from (10) of Definition 2.4 with $P=f \in C^{\infty}(M, \mathbb{R})$ and $P=X \in \Gamma(A)$, respectively [6]. The last equality is the dual of the third and holds as a consequence of the duality property for generalized Lie bialgebroids.

ii) The first equality was proved in [6] and the second also holds since it is the dual of the first.

iii) For any $f, g \in C^{\infty}(M, \mathbb{R})$ and $X \in \Gamma(A)$ we have

$\mathcal{L}_{d_{*}^{W} f}(g X)+\mathcal{L}_{* d^{\phi} f}^{W}(g X)=g\left(\mathcal{L}_{d_{*}^{W} f} X+\mathcal{L}_{* d^{\phi} f}^{W} X\right)+\left(\mathcal{L}_{d_{*}^{W} f}(g)+\mathcal{L}_{* d^{\phi} f}(g)\right)(X)$.

By ii), $\mathcal{L}_{d_{*}^{W} f}(g X)+\mathcal{L}_{* d^{\phi} f}^{W}(g X)=0$ and also $\mathcal{L}_{d_{*}^{W} f} X+\mathcal{L}_{* d^{\phi} f}^{W} X=0$. So, we obtain

$$
\mathcal{L}_{d_{*}^{W} f}(g)+\mathcal{L}_{* d^{\phi} f}(g)=0
$$

that is,

$$
\left\langle d g, d_{*}^{W} f\right\rangle+\left\langle d^{\phi} f, d_{*} g\right\rangle=0,
$$

or, equivalently,

$$
a\left(d_{*}^{W} f\right) g+a_{*}\left(d^{\phi} f\right) g=0 .
$$

Then, we conclude that

$$
\left(a \circ d_{*}^{W}+a_{*} \circ d^{\phi}\right) f=0 .
$$


iv) For any $f, g \in C^{\infty}(M, \mathbb{R})$,

$$
\begin{aligned}
\left\langle d^{\phi} f, d_{*}^{W} g\right\rangle+\left\langle d^{\phi} g, d_{*}^{W} f\right\rangle= & \left\langle d^{\phi} f, d_{*} g\right\rangle+\left\langle d g, d_{*}^{W} f\right\rangle \\
& +2 f g\langle\phi, W\rangle+g\left(a_{*}(\phi) f+a(W) f\right) .
\end{aligned}
$$

From i) and (29), we obtain $\left\langle d^{\phi} f, d_{*}^{W} g\right\rangle+\left\langle d^{\phi} g, d_{*}^{W} f\right\rangle=0$.

Lemma 3.6. Let $\left((A, \phi),\left(A^{*}, W\right)\right)$ be a generalized Lie bialgebroid over $M$. Then, for any $X \in \Gamma(A)$ and $\alpha \in \Gamma\left(A^{*}\right)$

$$
\left[a(X), a_{*}(\alpha)\right]=a_{*}\left(\mathcal{L}_{X}^{\phi} \alpha\right)-a\left(\mathcal{L}_{* \alpha}^{W} X\right)+a\left(d_{*}^{W}(\langle\alpha, X\rangle)\right) .
$$

Proof: For any $f \in C^{\infty}(M, \mathbb{R})$, we have

$$
\begin{aligned}
& {\left[a(X), a_{*}(\alpha)\right](f)-a_{*}\left(\mathcal{L}_{X}^{\phi} \alpha\right) f+a\left(\mathcal{L}_{* \alpha}^{W} X\right) f=} \\
& \quad=a(X)\left(\left\langle\alpha, d_{*} f\right\rangle\right)-\left\langle\mathcal{L}_{X}^{\phi} \alpha, d_{*} f\right\rangle-a_{*}(\alpha)\langle d f, X\rangle+\left\langle d f, \mathcal{L}_{* \alpha}^{W} X\right\rangle \\
& =\quad\left\langle\alpha,\left[X, d_{*} f\right]\right\rangle-\langle\phi, X\rangle\left(a_{*}(\alpha) f\right)-\left\langle[\alpha, d f]_{*}, X\right\rangle+\langle\alpha, X\rangle(a(X) f) \\
& \quad=\quad\left\langle\alpha,\left[X, d_{*}^{W} f\right]\right\rangle-f\langle\alpha,[X, W]\rangle+\left\langle\mathcal{L}_{* d f} \alpha, X\right\rangle-\langle\phi, X\rangle\left(a_{*}(\alpha) f\right) \\
& \stackrel{(26)}{=} \quad\left\langle\alpha, \mathcal{L}_{* d^{\phi} f}^{W} X\right\rangle-f\langle\alpha,[X, W]\rangle+\left\langle\mathcal{L}_{* d f} \alpha, X\right\rangle-\langle\phi, X\rangle\left(a_{*}(\alpha) f\right) \\
& \quad=\quad \mathcal{L}_{* d f}(\langle\alpha, X\rangle)+\langle\alpha, X\rangle(a(X) f)+f\langle\alpha, \underbrace{\mathcal{L}_{* \phi} X-[X, W]}_{=0}\rangle \\
& \quad=\mathcal{L}_{* d f}^{W}(\langle\alpha, X\rangle)=a\left(d_{*}^{W}\langle\alpha, X\rangle\right) f .
\end{aligned}
$$

\section{Proof of Theorem 3.4.}

First we notice that, since $\phi \in \Gamma\left(A^{*}\right)$ and $W \in \Gamma(A)$ are 1-cocycles of $A$ and $A^{*}$ respectively, and using i) of Lemma 3.5, a straightforward computation shows that, with $\theta=\phi+W$ and $\rho=a+a_{*}$,

$$
\left\langle\theta, \llbracket X_{1}+\alpha_{1}, X_{2}+\alpha_{2} \rrbracket\right\rangle=\rho\left(X_{1}+\alpha_{1}\right)\left\langle\theta, X_{2}+\alpha_{2}\right\rangle-\rho\left(X_{2}+\alpha_{2}\right)\left\langle\theta, X_{1}+\alpha_{1}\right\rangle
$$

holds for all $X_{1}+\alpha_{1}, X_{2}+\alpha_{2} \in \Gamma\left(A \oplus A^{*}\right)$.

Next, we show that the five conditions of Definition 3.1 hold.

1. The proof of condition 1 of Definition 3.1 involves a very long computation. We only give a short schedule, following the ideas of [16]. Let $e_{i}=X_{i}+\alpha_{i}$, 
$i=1,2,3$, be any sections of $A \oplus A^{*}$. Then,

$$
\begin{aligned}
\left(\llbracket e_{1}, e_{2} \rrbracket, e_{3}\right)_{+} & =\left[\frac { 1 } { 2 } \left(\left\langle\alpha_{3},\left[X_{1}, X_{2}\right]\right\rangle+\left\langle\left[\alpha_{1}, \alpha_{2}\right]_{*}, X_{3}\right\rangle+a\left(X_{3}\right)\left(e_{1}, e_{2}\right)_{-}\right.\right. \\
& \left.\left.-a_{*}\left(\alpha_{3}\right)\left(e_{1}, e_{2}\right)_{-}+\left(\left\langle\phi, X_{3}\right\rangle-\left\langle\alpha_{3}, W\right\rangle\right)\left(e_{1}, e_{2}\right)_{-}\right)+c . p .\right] \\
& +\frac{1}{2}\left(a\left(e_{1}\right)+a_{*}\left(e_{1}\right)\right)\left(e_{2}, e_{3}\right)_{+}-\frac{1}{2}\left(a\left(e_{2}\right)+a_{*}\left(e_{2}\right)\right)\left(e_{3}, e_{1}\right)_{+} \\
& +\frac{1}{2}\left(\left\langle\phi, X_{1}\right\rangle+\left\langle\alpha_{1}, W\right\rangle\right)\left(e_{2}, e_{3}\right)_{+} \\
& -\frac{1}{2}\left(\left\langle\phi, X_{2}\right\rangle+\left\langle\alpha_{2}, W\right\rangle\right)\left(e_{3}, e_{1}\right)_{+} \\
& =T\left(e_{1}, e_{2}, e_{3}\right)+\frac{1}{2} \rho\left(e_{1}\right)\left(e_{2}, e_{3}\right)_{+}-\frac{1}{2} \rho\left(e_{2}\right)\left(e_{3}, e_{1}\right)_{+} \\
& +\frac{1}{2}\left\langle\theta, e_{1}\right\rangle\left(e_{2}, e_{3}\right)_{+}-\frac{1}{2}\left\langle\theta, e_{2}\right\rangle\left(e_{3}, e_{1}\right)_{+} .
\end{aligned}
$$

Furthermore, we can prove the following equality:

$$
\begin{gathered}
\left(\llbracket e_{1}, e_{2} \rrbracket, e_{3}\right)_{-}+c . p .=T\left(e_{1}, e_{2}, e_{3}\right)+\left[\left(a\left(X_{3}\right)\left(e_{1}, e_{2}\right)_{-}+2 a_{*}\left(\alpha_{3}\right)\left(e_{1}, e_{2}\right)_{-}\right.\right. \\
\left.\left.-\left\langle\left[\alpha_{1}, \alpha_{2}\right]_{*}, X_{3}\right\rangle+\left(\left\langle\phi, X_{3}\right\rangle+2\left\langle\alpha_{3}, W\right\rangle\right)\left(e_{1}, e_{2}\right)_{-}\right)+c . p .\right]
\end{gathered}
$$

Let us set

$$
\llbracket \llbracket e_{1}, e_{2} \rrbracket, e_{3} \rrbracket+c \cdot p .=Y+\beta,
$$

where $Y$ (resp. $\beta$ ) stands for the part of $\llbracket \llbracket e_{1}, e_{2} \rrbracket, e_{3} \rrbracket+$ c.p. that belongs to $\Gamma(A)\left(\right.$ resp. $\left.\Gamma\left(A^{*}\right)\right)$. Using the formula $([5])$

$$
\mathcal{L}_{X}^{\phi} \circ \mathcal{L}_{Y}^{\phi}=\mathcal{L}_{[X, Y]}^{\phi}, \forall X, Y \in \Gamma(A),
$$

we deduce

$$
\begin{aligned}
\beta= & \left\{\left[\mathcal{L}_{X_{1}}^{\phi} \alpha_{2}-\mathcal{L}_{X_{2}}^{\phi} \alpha_{1}, \alpha_{3}\right]_{*}+\left[d^{\phi}\left(e_{1}, e_{2}\right)_{-}, \alpha_{3}\right]_{*}+\mathcal{L}_{\mathcal{L}_{* \alpha_{1}}^{W} X_{2}-\mathcal{L}_{* \alpha_{2}}^{W} X_{1}} \alpha_{3}\right. \\
& -\mathcal{L}_{d_{*}^{W}\left(e_{1}, e_{2}\right)_{-}}^{\phi} \alpha_{3}-\mathcal{L}_{X_{3}}^{\phi}\left[\alpha_{1}, \alpha_{2}\right]_{*}+d^{\phi}\left(\llbracket e_{1}, e_{2} \rrbracket, e_{3}\right)_{-} \\
& \left.-d^{\phi}\left(a\left(X_{3}\right)\left(e_{1}, e_{2}\right)_{-}\right)-d^{\phi}\left(\left\langle\phi, X_{3}\right\rangle\left(e_{1}, e_{2}\right)_{-}\right)\right\}+c . p . \\
= & \left\{d ^ { \phi } \left[\left(\llbracket e_{1}, e_{2} \rrbracket, e_{3}\right)_{-}-\left(a\left(X_{3}\right)\left(e_{1}, e_{2}\right)_{-}\right)-2\left(a_{*}\left(\alpha_{3}\right)\left(e_{1}, e_{2}\right)_{-}\right)\right.\right. \\
& \left.\left.+\left\langle\left[\alpha_{1}, \alpha_{2}\right]_{*}, X_{3}\right\rangle-\left(\left\langle\phi, X_{3}\right\rangle+2\left\langle\alpha_{3}, W\right\rangle\right)\left(e_{1}, e_{2}\right)_{-}\right)\right] \\
& -i_{X_{3}}\left(d^{\phi}\left[\alpha_{1}, \alpha_{2}\right]_{*}-\mathcal{L}_{* \alpha_{1}}^{W} d^{\phi} \alpha_{2}+\mathcal{L}_{* \alpha_{2}}^{W} d^{\phi} \alpha_{1}\right)-\left[d^{\phi}\left(e_{1}, e_{2}\right)_{-}, \alpha_{3}\right]_{*} \\
& \left.-\mathcal{L}_{d_{*}^{W}\left(e_{1}, e_{2}\right)_{-}}^{\phi} \alpha_{3}\right\}+c . p . .
\end{aligned}
$$


By Lemma 3.5 ii),

$$
\left[d^{\phi}\left(e_{1}, e_{2}\right)_{-}, \alpha_{3}\right]_{*}+\mathcal{L}_{d_{*}^{W}\left(e_{1}, e_{2}\right)_{-}}^{\phi} \alpha_{3}=0 .
$$

Moreover,

$$
\begin{aligned}
d^{\phi}\left[\alpha_{1}, \alpha_{2}\right]_{*}-\mathcal{L}_{* \alpha_{1}}^{W} d^{\phi} \alpha_{2}+\mathcal{L}_{* \alpha_{2}}^{W} d^{\phi} \alpha_{1} & =d^{\phi}\left[\alpha_{1}, \alpha_{2}\right]_{*}^{W}-\left[\alpha_{1}, d^{\phi} \alpha_{2}\right]_{*}^{W}+\left[\alpha_{2}, d^{\phi} \alpha_{1}\right]_{*}^{W} \\
& =0,
\end{aligned}
$$

by definition of generalized Lie bialgebroid.

So, we obtain

$$
\begin{aligned}
\beta= & d^{\phi}\left[\left(\llbracket e_{1}, e_{2} \rrbracket, e_{3}\right)_{-}-\left(a\left(X_{3}\right)\left(e_{1}, e_{2}\right)_{-}\right)-2\left(a_{*}\left(\alpha_{3}\right)\left(e_{1}, e_{2}\right)_{-}\right)+\left\langle\left[\alpha_{1}, \alpha_{2}\right]_{*}, X_{3}\right\rangle\right. \\
& \left.\left.-\left(\left\langle\phi, X_{3}\right\rangle+2\left\langle\alpha_{3}, W\right\rangle\right)\left(e_{1}, e_{2}\right)_{-}\right)\right]+c . p . \\
\stackrel{(31)}{=} & d^{\phi}\left(T\left(e_{1}, e_{2}, e_{3}\right)\right) .
\end{aligned}
$$

Similarly, one has

$$
Y=d_{*}^{W}\left(T\left(e_{1}, e_{2}, e_{3}\right)\right) .
$$

From (34) and (35), we conclude that

$$
\llbracket \llbracket e_{1}, e_{2} \rrbracket, e_{3} \rrbracket+c . p .=\mathcal{D}^{\theta} T\left(e_{1}, e_{2}, e_{3}\right) .
$$

2. For any $e_{1}=X_{1}+\alpha_{1}, e_{2}=X_{2}+\alpha_{2} \in \Gamma\left(A \oplus A^{*}\right)$, we compute

$$
\begin{aligned}
\rho\left(\llbracket X_{1}\right. & \left.+\alpha_{1}, X_{2}+\alpha_{2} \rrbracket\right)=a\left(\left[X_{1}, X_{2}\right]+\mathcal{L}_{* \alpha_{1}}^{W} X_{2}-\mathcal{L}_{* \alpha_{2}}^{W} X_{1}-d_{*}^{W}\left(e_{1}, e_{2}\right)_{-}\right) \\
& +a_{*}\left(\left[\alpha_{1}, \alpha_{2}\right]_{*}+\mathcal{L}_{X_{1}}^{\phi} \alpha_{2}-\mathcal{L}_{X_{2}}^{\phi} \alpha_{1}+d^{\phi}\left(e_{1}, e_{2}\right)_{-}\right) \\
= & {\left[a\left(X_{1}\right), a\left(X_{2}\right)\right]+\left[a_{*}\left(\alpha_{1}\right), a_{*}\left(\alpha_{2}\right)\right] } \\
& +\left\{a\left(\mathcal{L}_{* \alpha_{1}}^{W} X_{2}\right)-a_{*}\left(\mathcal{L}_{X_{2}}^{\phi} \alpha_{1}\right)-\frac{1}{2} a\left(d_{*}^{W}\left\langle\alpha_{1}, X_{2}\right\rangle\right)+\frac{1}{2} a_{*}\left(d^{\phi}\left\langle\alpha_{1}, X_{2}\right\rangle\right)\right\} \\
& -\left\{a\left(\mathcal{L}_{* \alpha_{2}}^{W} X_{1}\right)-a_{*}\left(\mathcal{L}_{X_{1}}^{\phi} \alpha_{2}\right)-\frac{1}{2} a\left(d_{*}^{W}\left\langle\alpha_{2}, X_{1}\right\rangle\right)+\frac{1}{2} a_{*}\left(d^{\phi}\left\langle\alpha_{2}, X_{1}\right\rangle\right)\right\} \\
\stackrel{(27)}{=} & {\left[a\left(X_{1}\right), a\left(X_{2}\right)\right]+\left[a_{*}\left(\alpha_{1}\right), a_{*}\left(\alpha_{2}\right)\right] } \\
+ & \left\{a\left(\mathcal{L}_{* \alpha_{1}}^{W} X_{2}\right)-a_{*}\left(\mathcal{L}_{X_{2}}^{\phi} \alpha_{1}\right)-a\left(d_{*}^{W}\left\langle\alpha_{1}, X_{2}\right\rangle\right)\right\} \\
& -\left\{a\left(\mathcal{L}_{* \alpha_{2}}^{W} X_{1}\right)-a_{*}\left(\mathcal{L}_{X_{1}}^{\phi} \alpha_{2}\right)-a\left(d_{*}^{W}\left\langle\alpha_{2}, X_{1}\right\rangle\right)\right\} .
\end{aligned}
$$


On the other hand,

$$
\begin{aligned}
{\left[\rho\left(X_{1}+\alpha_{1}\right), \rho\left(X_{2}+\alpha_{2}\right)\right]=} & {\left[a\left(X_{1}\right), a\left(X_{2}\right)\right]+\left[a_{*}\left(\alpha_{1}\right), a_{*}\left(\alpha_{2}\right)\right] } \\
& +\left[a\left(X_{1}\right), a_{*}\left(\alpha_{2}\right)\right]-\left[a\left(X_{2}\right), a_{*}\left(\alpha_{1}\right)\right]
\end{aligned}
$$

and, by Lemma 3.6, we conclude that $\left[\rho\left(X_{1}+\alpha_{1}\right), \rho\left(X_{2}+\alpha_{2}\right)\right]=\left[\rho\left(X_{1}+\right.\right.$ $\left.\left.\alpha_{1}\right), \rho\left(X_{2}+\alpha_{2}\right)\right]$.

3. For any $e_{1}=X_{1}+\alpha_{1}, e_{2}=X_{2}+\alpha_{2} \in \Gamma\left(A \oplus A^{*}\right)$ and $f \in C^{\infty}(M, \mathbb{R})$, we have

$$
\begin{aligned}
\llbracket X_{1}+ & \alpha_{1}, f\left(X_{2}+\alpha_{2}\right) \rrbracket= \\
& \left(\left[X_{1}, f X_{2}\right]^{\phi}+\mathcal{L}_{* \alpha_{1}}^{W}\left(f X_{2}\right)-\mathcal{L}_{*\left(f \alpha_{2}\right)}^{W} X_{1}-d_{*}^{W}\left(f\left(e_{1}, e_{2}\right)_{-}\right)\right) \\
& +\left(\left[\alpha_{1}, f \alpha_{2}\right]_{*}^{W}+\mathcal{L}_{X_{1}}^{\phi}\left(f \alpha_{2}\right)-\mathcal{L}_{f X_{2}}^{\phi} \alpha_{1}+d^{\phi}\left(f\left(e_{1}, e_{2}\right)_{-}\right)\right) \\
= & \left\{f\left(\left[X_{1}, X_{2}\right]+\mathcal{L}_{* \alpha_{1}}^{W} X_{2}-\mathcal{L}_{* \alpha_{2}}^{W} X_{1}-d_{*}^{W}\left(e_{1}, e_{2}\right)_{-}\right)\right. \\
& \left.+\left(a\left(X_{1}\right) f\right) X_{2}+\left(a_{*}\left(\alpha_{1}\right) f\right) X_{2}-\left(\left\langle\alpha_{2}, X_{1}\right\rangle+\left(e_{1}, e_{2}\right)_{-}\right) d_{*} f\right\} \\
& +\left\{f\left(\left[\alpha_{1}, \alpha_{2}\right]_{*}^{W}+\mathcal{L}_{X_{1}}^{\phi} \alpha_{2}-\mathcal{L}_{X_{2}}^{\phi} \alpha_{1}+d^{\phi}\left(e_{1}, e_{2}\right)_{-}\right)\right. \\
& \left.+\left(a\left(X_{1}\right) f\right) \alpha_{2}+\left(a_{*}\left(\alpha_{1}\right) f\right) \alpha_{2}+\left(\left(e_{1}, e_{2}\right)_{-}-\left\langle\alpha_{1}, X_{2}\right\rangle\right) d f\right\} \\
= & f \llbracket X_{1}+\alpha_{1}, X_{2}+\alpha_{2} \rrbracket \\
& +\left(\left(a\left(X_{1}\right)+a_{*}\left(\alpha_{1}\right)\right) f\right)\left(X_{2}+\alpha_{2}\right)-\left(e_{1}, e_{2}\right)_{+}\left(d_{*} f+d f\right) .
\end{aligned}
$$

4. For any $f, g \in C^{\infty}(M, \mathbb{R})$, we have

$$
\left(\mathcal{D}^{\theta} f, \mathcal{D}^{\theta} g\right)_{+}=\frac{1}{2}\left(\left\langle d^{\phi} f, d_{*}^{W} g\right\rangle+\left\langle d^{\phi} g, d_{*}^{W} f\right\rangle\right) \stackrel{(28)}{=} 0 .
$$

5. For any sections $e_{1}=X_{1}+\alpha_{1}, e_{2}=X_{2}+\alpha_{2}$ and $h=Y+\beta$ of $A \oplus A^{*}$, we compute

$\rho(h)\left(e_{1}, e_{2}\right)_{+}=\frac{1}{2} a(Y)\left(\left\langle\alpha_{1}, X_{2}\right\rangle+\left\langle\alpha_{2}, X_{1}\right\rangle\right)+\frac{1}{2} a_{*}(\beta)\left(\left\langle\alpha_{1}, X_{2}\right\rangle+\left\langle\alpha_{2}, X_{1}\right\rangle\right)$

and, taking account that

$$
\left(h, e_{1}\right)_{+}+\left(h, e_{1}\right)_{-}=\left\langle\beta, X_{1}\right\rangle \text { and }\left(h, e_{1}\right)_{+}-\left(h, e_{1}\right)_{-}=\left\langle\alpha_{1}, Y\right\rangle,
$$




$$
\begin{aligned}
\left(\llbracket h, e_{1} \rrbracket+\mathcal{D}^{\theta}\left(h, e_{1}\right)_{+}, e_{2}\right)_{+} & =\frac{1}{2}\left(\left\langle\left[\beta, \alpha_{1}\right]_{*}^{W}+\mathcal{L}_{Y}^{\phi} \alpha_{1}-\mathcal{L}_{X_{1}}^{\phi} \beta+d^{\phi}\left(\left\langle\beta, X_{1}\right\rangle\right), X_{2}\right\rangle\right. \\
& \left.+\left\langle\alpha_{2},\left[Y, X_{1}\right]^{\phi}+\mathcal{L}_{* \beta}^{W} X_{1}-\mathcal{L}_{* \alpha_{1}}^{W} Y+d_{*}^{W}\left(\left\langle\alpha_{1}, Y\right\rangle\right)\right\rangle\right)
\end{aligned}
$$

Similarly,

$$
\begin{aligned}
\left(e_{1}, \llbracket h, e_{2} \rrbracket+\mathcal{D}^{\theta}\left(h, e_{2}\right)_{+}\right)_{+} & =\frac{1}{2}\left(\left\langle\left[\beta, \alpha_{2}\right]_{*}^{W}+\mathcal{L}_{Y}^{\phi} \alpha_{2}-\mathcal{L}_{X_{2}}^{\phi} \beta+d^{\phi}\left(\left\langle\beta, X_{2}\right\rangle\right), X_{1}\right\rangle\right. \\
& \left.+\left\langle\alpha_{1},\left[Y, X_{2}\right]^{\phi}+\mathcal{L}_{* \beta}^{W} X_{2}-\mathcal{L}_{* \alpha_{2}}^{W} Y+d_{*}^{W}\left(\left\langle\alpha_{2}, Y\right\rangle\right)\right\rangle\right) .
\end{aligned}
$$

Adding up (37) and (38) we obtain, using the equality $\left\langle\mathcal{L}_{X_{1}}^{\phi} \beta, X_{2}\right\rangle=$ $i_{X_{2}}\left(i_{X_{1}} d^{\phi} \beta+d^{\phi} i_{X_{1}} \beta\right)$ and its dual version,

$$
\begin{aligned}
\left(\llbracket h, e_{1} \rrbracket+\mathcal{D}^{\theta}\left(h, e_{1}\right)_{+}, e_{2}\right)_{+}+\left(e_{1}, \llbracket h, e_{2} \rrbracket+\mathcal{D}^{\theta}\left(h, e_{2}\right)_{+}\right)_{+}= \\
=\frac{1}{2}\left\{\mathcal{L}_{Y}^{\phi}\left(\left\langle\alpha_{1}, X_{2}\right\rangle\right)-i_{X_{2}}\left(i_{X_{1}} d^{\phi} \beta\right)+\mathcal{L}_{* \beta}^{W}\left(\left\langle\alpha_{2}, X_{1}\right\rangle\right)-i_{\alpha_{2}}\left(i_{\alpha_{1}} d_{*}^{W} Y\right)\right. \\
\left.\quad+\mathcal{L}_{Y}^{\phi}\left(\left\langle\alpha_{2}, X_{1}\right\rangle\right)-i_{X_{1}}\left(i_{X_{2}} d^{\phi} \beta\right)+\mathcal{L}_{* \beta}^{W}\left(\left\langle\alpha_{1}, X_{2}\right\rangle\right)-i_{\alpha_{1}}\left(i_{\alpha_{2}} d_{*}^{W} Y\right)\right\} \\
=\frac{1}{2}\left\{a(Y)\left(\left\langle\alpha_{1}, X_{2}\right\rangle+\left\langle\alpha_{2}, X_{1}\right\rangle\right)+\langle\phi, Y\rangle\left(\left\langle\alpha_{1}, X_{2}\right\rangle+\left\langle\alpha_{2}, X_{1}\right\rangle\right)\right. \\
\left.\quad+a_{*}(\beta)\left(\left\langle\alpha_{1}, X_{2}\right\rangle+\left\langle\alpha_{2}, X_{1}\right\rangle\right)+\langle\beta, W\rangle\left(\left\langle\alpha_{1}, X_{2}\right\rangle+\left\langle\alpha_{2}, X_{1}\right\rangle\right)\right\} \\
=\rho(h)\left(e_{1}, e_{2}\right)_{+}+\langle\theta, h\rangle\left(e_{1}, e_{2}\right)_{+} .
\end{aligned}
$$

The first example of a generalized Courant algebroid which is a double of a generalized Lie bialgebroid, comes from the Jacobi manifolds. As it is illustrated by Example 2.6, we can associate with each Jacobi manifold $(M, \Lambda, E)$ a generalized Lie bialgebroid

$$
\left(\left(\left(T^{*} M \times \mathbb{R},[., .]_{(\Lambda, E)}, \pi \circ(\Lambda, E)^{\#}\right),(-E, 0)\right),((T M \times \mathbb{R},[., .], \pi),(0,1))\right) .
$$

Let us denote by $\mathcal{E}^{1}(M)$ the vector bundle over $M,(T M \times \mathbb{R}) \oplus\left(T^{*} M \times\right.$ $\mathbb{R}) \rightarrow M$. The next proposition is an immediate consequence of Theorem 3.4 . 
Proposition 3.7. If $(M, \Lambda, E)$ is a Jacobi manifold, then the pair $\left(\mathcal{E}^{1}(M), \theta\right)$, with $\theta=(0,1)+(-E, 0) \in \Gamma\left(\left(\mathcal{E}^{1}(M)\right)^{*}\right)$ is a generalized Courant algebroid.

For this generalized Courant algebroid $\left(\mathcal{E}^{1}(M), \theta\right)$, the bracket (23) is given, for any sections $e_{1}=\left(X_{1}, f_{1}\right)+\left(\alpha_{1}, g_{1}\right)$ and $e_{2}=\left(X_{2}, f_{2}\right)+\left(\alpha_{2}, g_{2}\right)$ of $\mathcal{E}^{1}(M)$, by:

$$
\begin{aligned}
& \llbracket\left(X_{1}, f_{1}\right)+\left(\alpha_{1}, g_{1}\right),\left(X_{2}, f_{2}\right)+\left(\alpha_{2}, g_{2}\right) \rrbracket= \\
&=\left(\left[\left(X_{1}, f_{1}\right),\left(X_{2}, f_{2}\right)\right]^{(0,1)}+\mathcal{L}_{*\left(\alpha_{1}, g_{1}\right)}^{(-E, 0)}\left(X_{2}, f_{2}\right)-\mathcal{L}_{*\left(\alpha_{2}, g_{2}\right)}^{(-E, 0)}\left(X_{1}, f_{1}\right)\right. \\
&\left.-d_{*}^{(-E, 0)}\left(e_{1}, e_{2}\right)_{-}\right) \\
&+\left(\left[\left(\alpha_{1}, g_{1}\right),\left(\alpha_{2}, g_{2}\right)\right]_{(\Lambda, E)}+\mathcal{L}_{\left(X_{1}, f_{1}\right)}^{(0,1)}\left(\alpha_{2}, g_{2}\right)-\mathcal{L}_{\left(X_{2}, f_{2}\right)}^{(0,1)}\left(\alpha_{1}, g_{1}\right)\right. \\
&\left.+d^{(0,1)}\left(e_{1}, e_{2}\right)_{-}\right)
\end{aligned}
$$

where

$$
\begin{aligned}
\left(e_{1}, e_{2}\right)_{-} & =\frac{1}{2}\left\{\left\langle\left(\alpha_{1}, g_{1}\right),\left(X_{2}, f_{2}\right)\right\rangle-\left\langle\left(\alpha_{2}, g_{2}\right),\left(X_{1}, f_{1}\right)\right\rangle\right\} \\
& =\frac{1}{2}\left\{\left\langle\alpha_{1}, X_{2}\right\rangle-\left\langle\alpha_{2}, X_{1}\right\rangle+f_{2} g_{1}-f_{1} g_{2}\right\} .
\end{aligned}
$$

Besides the skew-symmetric bilinear form $(., .)_{-}$in $\Gamma\left(\mathcal{E}^{1}(M)\right)$ given by (41), there exists a symmetric bilinear form in $\Gamma\left(\mathcal{E}^{1}(M)\right)$ :

$$
\left(e_{1}, e_{2}\right)_{+}=\frac{1}{2}\left\{\left\langle\alpha_{1}, X_{2}\right\rangle+\left\langle\alpha_{2}, X_{1}\right\rangle+f_{2} g_{1}+f_{1} g_{2}\right\} .
$$

Let us now consider the Lie algebroid $(T M \times \mathbb{R},[.,],. \pi)$ and its 1-cocycle $\phi=(0,1) \in \Gamma\left(T^{*} M \times \mathbb{R}\right)$ (see (13)). Its dual vector bundle $T^{*} M \times \mathbb{R}$ is also a Lie algebroid if we endow the space of sections with an abelian Lie algebra structure and take the null anchor map; that is $[.,]_{*}=0$ and $\rho_{*}=0$. Moreover, the section $W=(0,0)$ of $T M \times \mathbb{R}$ is obviously a 1-cocycle for the Lie algebroid $T^{*} M \times \mathbb{R}$ and, from Definitions 2.4 or 2.5 , it is easy immediate to see that the pair $\left((T M \times \mathbb{R},(0,1)),\left(T^{*} M \times \mathbb{R},(0,0)\right)\right)$ is a generalized Lie bialgebroid. Therefore, by Theorem 3.4, we conclude:

Corollary 3.8. The pair $\left(\mathcal{E}^{1}(M), \psi\right)$, with $\psi=((0,1)+(0,0)) \in \Gamma\left(\left(\mathcal{E}^{1}(M)\right)^{*}\right)$, is a generalized Courant algebroid. 
The explicit expression of the bracket (23) on the space of sections of the generalized Courant algebroid of Corollary 3.8 is the following:

$$
\begin{aligned}
& \llbracket\left(X_{1}, f_{1}\right)+\left(\alpha_{1}, g_{1}\right),\left(X_{2}, f_{2}\right)+\left(\alpha_{2}, g_{2}\right) \rrbracket=\left[\left(X_{1}, f_{1}\right),\left(X_{2}, f_{2}\right)\right]^{(0,1)} \\
& +\left(\mathcal{L}_{\left(X_{1}, f_{1}\right)}^{(0,1)}\left(\alpha_{2}, g_{2}\right)-\mathcal{L}_{\left(X_{2}, f_{2}\right)}^{(0,1)}\left(\alpha_{1}, g_{1}\right)+d^{(0,1)}\left(e_{1}, e_{2}\right)_{-}\right),
\end{aligned}
$$

with $e_{i}=\left(X_{i}, f_{i}\right)+\left(\alpha_{i}, g_{i}\right), i=1,2$, any sections of $\mathcal{E}^{1}(M)$.

Or, a simple computation gives

$$
\begin{aligned}
\mathcal{L}_{\left(X_{1}, f_{1}\right)}^{(0,1)}\left(\alpha_{2}, g_{2}\right) & =\left\langle(0,1),\left(X_{1}, f_{1}\right)\right\rangle\left(\alpha_{2}, g_{2}\right)+i_{\left(X_{1}, f_{1}\right)} d\left(\alpha_{2}, g_{2}\right)+d\left(i_{\left(X_{1}, f_{1}\right)}\left(\alpha_{2}, g_{2}\right)\right) \\
& =\left(f_{1} \alpha_{2}, f_{1} g_{2}\right)+i_{\left(X_{1}, f_{1}\right)}\left(\delta \alpha_{2},-\delta g_{2}\right)+d\left(i_{X_{1}} \alpha_{2}+f_{1} g_{2}, 0\right) \\
& =\left(f_{1} \alpha_{2}+\mathcal{L}_{X_{1}} \alpha_{2}+g_{2} \delta f_{1}, f_{1} g_{2}+X_{1}\left(g_{2}\right)\right)
\end{aligned}
$$

and, analogously,

$$
\mathcal{L}_{\left(X_{2}, f_{2}\right)}^{(0,1)}\left(\alpha_{1}, g_{1}\right)=\left(f_{2} \alpha_{1}+\mathcal{L}_{X_{2}} \alpha_{1}+g_{1} \delta f_{2}, f_{2} g_{1}+X_{2}\left(g_{1}\right)\right) .
$$

We also compute

$$
\begin{aligned}
d^{(0,1)}\left(e_{1}, e_{2}\right)_{-}= & d\left(e_{1}, e_{2}\right)_{-}+\left(0,\left(e_{1}, e_{2}\right)_{-}\right) \\
= & \left(\frac{1}{2} \delta\left(\left\langle\alpha_{1}, X_{2}\right\rangle-\left\langle\alpha_{2}, X_{1}\right\rangle+f_{2} g_{1}-f_{1} g_{2}\right),\right. \\
& \left.\frac{1}{2}\left(\left\langle\alpha_{1}, X_{2}\right\rangle-\left\langle\alpha_{2}, X_{1}\right\rangle+f_{2} g_{1}-f_{1} g_{2}\right)\right) .
\end{aligned}
$$

So, by (44), (45) and (46), we obtain

$$
\begin{aligned}
\llbracket\left(X_{1}, f_{1}\right)+\left(\alpha_{1}, g_{1}\right),\left(X_{2}, f_{2}\right)+\left(\alpha_{2}, g_{2}\right) \rrbracket= \\
=\left(\left[X_{1}, X_{2}\right], X_{1}\left(f_{2}\right)-X_{2}\left(f_{1}\right)\right)+ \\
\quad+\left(f_{1} \alpha_{2}-f_{2} \alpha_{1}+\mathcal{L}_{X_{1}} \alpha_{2}-\mathcal{L}_{X_{2}} \alpha_{1}+\frac{1}{2} \delta\left(\left\langle\alpha_{1}, X_{2}\right\rangle-\left\langle\alpha_{2}, X_{1}\right\rangle\right)\right. \\
\quad+\frac{1}{2}\left(f_{2} \delta g_{1}-g_{1} \delta f_{2}+g_{2} \delta f_{1}-f_{1} \delta g_{2}\right), \\
\left.\quad X_{1}\left(g_{2}\right)-X_{2}\left(g_{1}\right)+\frac{1}{2}\left(\left\langle\alpha_{1}, X_{2}\right\rangle-\left\langle\alpha_{2}, X_{1}\right\rangle\right)+\frac{1}{2}\left(f_{1} g_{2}-f_{2} g_{1}\right)\right) .
\end{aligned}
$$


This is exactly the bracket introduced in the space of sections of $\mathcal{E}^{1}(M)$ by A. Wade in [23]. Moreover, as it was expected, contents of Proposition 3.3 in [23] expresses conditions 1 and 3 of our Definition 3.1 of generalized Courant algebroid, for the particular case of $\left(\mathcal{E}^{1}(M),(0,1)+(0,0)\right)$. If $L \subset \mathcal{E}^{1}(M)$ is a Dirac structure for the generalized Courant algebroid $\left(\mathcal{E}^{1}(M),(0,1)+(0,0)\right)$, then by Proposition 3.3, $\left(L,\left.\rho\right|_{L},\left.[.,]\right|_{L}.\right)$ is a Lie algebroid over $M$; and this is the contents of Theorem 3.4 in [23]. Finally, also by Proposition 3.3, we have that $(0,1)+(0,0) \in \Gamma\left(L^{*}\right)$ is a 1-cocycle for the Lie algebroid $\left(L,\left.\rho\right|_{L},\left.[.,]\right|_{L}.\right)$. This fact was pointed out in [7].

At this point we can make the following analogy: likewise the bracket introduced by Courant in [2] is obtained from the bracket in the double $T M \oplus$ $T^{*} M$ of the Lie bialgebroid $\left(T M, T^{*} M\right)([18])$ in the particular case where $T^{*} M$ is endowed with the null Lie algebroid structure, the bracket introduced by Wade in [23] is obtained from the bracket (23) in the double $\mathcal{E}^{1}(M)$ of the generalized Lie bialgebroid $\left((T M \times \mathbb{R},(0,1)),\left(T^{*} M \times \mathbb{R},(0,0)\right)\right)$, in the particular case where $T^{*} M \times \mathbb{R}$ is endowed with the null Lie algebroid structure.

\section{Dirac structures for generalized Courant algebroids}

In this section we present some examples of Dirac structures for generalized Courant algebroids which are graphs of bivector fields.

Let $\left((A, \phi),\left(A^{*}, W\right)\right)$ be a generalized Lie bialgebroid over $M$ and $\Omega$ a $A$ bivector field, i.e. $\Omega \in \Gamma\left(\bigwedge^{2} A\right)$. Let us denote by $\Omega^{\#}$ the associated vector bundle map, $\Omega^{\#}: A^{*} \rightarrow A$, and by $L$ the graph of $\Omega^{\#}$, considered as a subbundle of $A \oplus A^{*}$,

$$
L=\left\{\Omega^{\#} \alpha+\alpha, \alpha \in A^{*}\right\}
$$

Proposition 4.1. The graph of $\Omega^{\#}$ is a Dirac structure for the generalized Courant algebroid $\left(A \oplus A^{*}, \phi+W\right)$ if and only if the Maurer-Cartan type equation

$$
d_{*}^{W} \Omega+\frac{1}{2}[\Omega, \Omega]^{\phi}=0
$$

holds. 
Proof: If $\Omega^{\#} \alpha+\alpha, \Omega^{\#} \beta+\beta \in L=$ graph $\Omega^{\#}$ then, since $\Omega$ is skew-symmetric, we have

$$
\left(\Omega^{\#} \alpha+\alpha, \Omega^{\#} \beta+\beta\right)_{+}=\frac{1}{2}\left(\left\langle\alpha, \Omega^{\#} \beta\right\rangle+\left\langle\beta, \Omega^{\#} \alpha\right\rangle\right)=0,
$$

and $L$ is a maximal isotropic subbundle of $A \oplus A^{*}$.

It remains to show that $L$ is integrable. Or the bracket (23) expresses, in this case, for any sections $\Omega^{\#} \alpha+\alpha$ and $\Omega^{\#} \beta+\beta$ of $L$, as follows:

$$
\begin{aligned}
\llbracket \Omega^{\#} \alpha+\alpha, \Omega^{\#} \beta+\beta \rrbracket= & \left(\left[\Omega^{\#} \alpha, \Omega^{\#} \beta\right]+\mathcal{L}_{* \alpha}^{W}\left(\Omega^{\#} \beta\right)-\mathcal{L}_{* \beta}^{W}\left(\Omega^{\#} \alpha\right)+d_{*}^{W}(\Omega(\alpha, \beta))\right) \\
& +\left([\alpha, \beta]_{*}^{W}+\mathcal{L}_{\Omega^{\#} \alpha}^{\phi} \beta-\mathcal{L}_{\Omega^{\#} \beta}^{\phi} \alpha-d^{\phi}(\Omega(\alpha, \beta))\right) .
\end{aligned}
$$

If we denote by $[\alpha, \beta]_{\Omega}$ the last three terms of (50),

$$
[\alpha, \beta]_{\Omega}=\mathcal{L}_{\Omega \# \alpha}^{\phi} \beta-\mathcal{L}_{\Omega^{\#} \beta}^{\phi} \alpha-d^{\phi}(\Omega(\alpha, \beta)),
$$

then, $L$ is integrable if and only if

$$
\left[\Omega^{\#} \alpha, \Omega^{\#} \beta\right]+\mathcal{L}_{* \alpha}^{W}\left(\Omega^{\#} \beta\right)-\mathcal{L}_{* \beta}^{W}\left(\Omega^{\#} \alpha\right)+d_{*}^{W}(\Omega(\alpha, \beta))=\Omega^{\#}\left([\alpha, \beta]_{*}+[\alpha, \beta]_{\Omega}\right) .
$$

For any $\alpha, \beta \in \Gamma\left(A^{*}\right)$, a straightforward computation leads to

$$
\left(d_{*}^{W} \Omega\right)(\alpha, \beta)=\mathcal{L}_{* \alpha}^{W}\left(\Omega^{\#} \beta\right)-\mathcal{L}_{* \beta}^{W}\left(\Omega^{\#} \alpha\right)+d_{*}^{W}(\Omega(\alpha, \beta))-\Omega^{\#}\left([\alpha, \beta]_{*}\right) ;
$$

and so (52) is equivalent to

$$
\left[\Omega^{\#} \alpha, \Omega^{\#} \beta\right]+\left(d_{*}^{W} \Omega\right)(\alpha, \beta)=\Omega^{\#}\left([\alpha, \beta]_{\Omega}\right) .
$$

On the other hand, for any $A$-bivector field $\Omega$, the following formula holds (see [12]):

$$
\left[\Omega^{\#} \alpha, \Omega^{\#} \beta\right]=\Omega^{\#}\left(\mathcal{L}_{\Omega \# \alpha} \beta-\mathcal{L}_{\Omega \# \beta} \alpha-d(\Omega(\alpha, \beta))\right)+\frac{1}{2}[\Omega, \Omega](\alpha, \beta)
$$

and from (8),

$$
\frac{1}{2}[\Omega, \Omega]^{\phi}(\alpha, \beta)=\frac{1}{2}[\Omega, \Omega](\alpha, \beta)+\left(\Omega^{\#} \phi \wedge \Omega\right)(\alpha, \beta) .
$$

Using (55) and (56) in (54), we conclude that $L$ is integrable if and only if 


$$
\begin{gathered}
\Omega^{\#}\left(\mathcal{L}_{\Omega^{\#} \alpha} \beta-\mathcal{L}_{\Omega^{\#} \beta} \alpha-d(\Omega(\alpha, \beta))\right)+\frac{1}{2}[\Omega, \Omega]^{\phi}(\alpha, \beta) \\
-\left(\left(\Omega^{\#} \phi\right) \wedge \Omega\right)(\alpha, \beta)+\left(d_{*}^{W} \Omega\right)(\alpha, \beta)=\Omega^{\#}\left([\alpha, \beta]_{\Omega}\right)
\end{gathered}
$$

that is, if and only if,

$$
\begin{aligned}
& \left(d_{*}^{W} \Omega\right)(\alpha, \beta)+\frac{1}{2}[\Omega, \Omega]^{\phi}(\alpha, \beta)= \\
& =\Omega^{\#}\left([\alpha, \beta]_{\Omega}\right)-\underbrace{\Omega^{\#}\left(\mathcal{L}_{\Omega^{\#} \alpha} \beta-\mathcal{L}_{\Omega^{\#} \beta} \alpha-d(\Omega(\alpha, \beta))\right)+\left(\left(\Omega^{\#} \phi\right) \wedge \Omega\right)(\alpha, \beta)}_{=\Omega^{\#}([\alpha, \beta] \Omega)} \\
& =0 .
\end{aligned}
$$

Let us now consider the generalized Courant algebroid $\left(\mathcal{E}^{1}(M), \psi\right)$, with $\psi=((0,1)+(0,0))$, treated in Corollary 3.8. A section of $\bigwedge^{2}(T M \times \mathbb{R})$ is, in this case, a pair $(\Lambda, E)$ where $\Lambda$ and $E$ are, respectively, a bivector field and a vector field on $M$. The graph of $(\Lambda, E)^{\#}$ is a subbundle $L$ of $\mathcal{E}^{1}(M)$ whose space of sections is

$$
\begin{aligned}
\Gamma(L)= & \left\{(\Lambda, E)^{\#}(\alpha, g)+(\alpha, g),(\alpha, g) \in \Omega^{1}(M) \times C^{\infty}(M, \mathbb{R})\right\} \\
& \stackrel{(2)}{=}\left\{\left(\Lambda^{\#} \alpha+g E,-\langle\alpha, E\rangle\right)+(\alpha, g),(\alpha, g) \in \Omega^{1}(M) \times C^{\infty}(M, \mathbb{R})\right\} .
\end{aligned}
$$

By Proposition 4.1, $L$ is a Dirac structure for the generalized Courant algebroid $\left(\mathcal{E}^{1}(M), \psi\right)$ if and only if

$$
\underbrace{d_{*}^{(0,0)}(\Lambda, E)}_{=0}+\frac{1}{2}[(\Lambda, E),(\Lambda, E)]^{(0,1)}=0 \Leftrightarrow[(\Lambda, E),(\Lambda, E)]^{(0,1)}=0 .
$$

But $[(\Lambda, E),(\Lambda, E)]^{(0,1)}=0$ if and only if $(M, \Lambda, E)$ is a Jacobi manifold (see [6]). So, we obtain a characterization of Jacobi manifolds in terms of Dirac structures and we recover a result from [23]: The graph of $(\Lambda, E)$ is a Dirac structure for $\left(\mathcal{E}^{1}(M), \psi\right)$ if and only if $(\Lambda, E)$ is a Jacobi structure on $M$.

We recall that two Jacobi structures $(\Lambda, E)$ and $\left(\Lambda^{\prime}, E^{\prime}\right)$ on a manifold $M$ are said to be compatible if their sum is still a Jacobi structure on $M$, [20]. 
Proposition 4.2. Let $(M, \Lambda, E)$ be a Jacobi manifold and $\left(\mathcal{E}^{1}(M), \theta\right)$, with $\theta=(0,1)+(-E, 0) \in \Gamma\left(\left(\mathcal{E}^{1}(M)\right)^{*}\right)$ the generalized Courant algebroid associated, as in Proposition 3.7, and let $\left(\Lambda^{\prime}, E^{\prime}\right)$ be a section of $\Lambda^{2}(T M \times \mathbb{R})$. Then, the pair $\left(\Lambda+\Lambda^{\prime}, E+E^{\prime}\right)=(\Lambda, E)+\left(\Lambda^{\prime}, E^{\prime}\right)$ determines a Jacobi structure on $M$ if and only if

$$
d_{*}^{(-E, 0)}\left(\Lambda^{\prime}, E^{\prime}\right)+\frac{1}{2}\left[\left(\Lambda^{\prime}, E^{\prime}\right),\left(\Lambda^{\prime}, E^{\prime}\right)\right]^{(0,1)}=0 .
$$

Moreover, $\left(\Lambda^{\prime}, E^{\prime}\right)$ is a Jacobi structure on $M$, compatible with $(\Lambda, E)$, if and only if

$$
d_{*}^{(-E, 0)}\left(\Lambda^{\prime}, E^{\prime}\right)=0 \quad \text { and } \quad\left[\left(\Lambda^{\prime}, E^{\prime}\right),\left(\Lambda^{\prime}, E^{\prime}\right)\right]^{(0,1)}=0 .
$$

Proof: As we have already remarked, $(\Lambda, E)+\left(\Lambda^{\prime}, E^{\prime}\right)$ determines a Jacobi structure on $M$ if and only if

$$
\left[(\Lambda, E)+\left(\Lambda^{\prime}, E^{\prime}\right),(\Lambda, E)+\left(\Lambda^{\prime}, E^{\prime}\right)\right]^{(0,1)}=0,
$$

or equivalently, if and only if

$$
2\left[(\Lambda, E),\left(\Lambda^{\prime}, E^{\prime}\right)\right]^{(0,1)}+\left[\left(\Lambda^{\prime}, E^{\prime}\right),\left(\Lambda^{\prime}, E^{\prime}\right)\right]^{(0,1)}=0 .
$$

But, since $d_{*}^{(-E, 0)}\left(\Lambda^{\prime}, E^{\prime}\right)=\left[(\Lambda, E),\left(\Lambda^{\prime}, E^{\prime}\right)\right]^{(0,1)}$ (see $\left.[6]\right)$, the equation (61) turns to

$$
d_{*}^{(-E, 0)}\left(\Lambda^{\prime}, E^{\prime}\right)+\frac{1}{2}\left[\left(\Lambda^{\prime}, E^{\prime}\right),\left(\Lambda^{\prime}, E^{\prime}\right)\right]^{(0,1)}=0 .
$$

For the last assertion, we have that $\left(\Lambda^{\prime}, E^{\prime}\right)$ is a Jacobi structure on $M$ if and only if $\left[\left(\Lambda^{\prime}, E^{\prime}\right),\left(\Lambda^{\prime}, E^{\prime}\right)\right]^{(0,1)}=0$. Moreover,

$d_{*}^{(-E, 0)}\left(\Lambda^{\prime}, E^{\prime}\right)=0 \quad \Longleftrightarrow \quad\left[\Lambda, \Lambda^{\prime}\right]+E \wedge \Lambda^{\prime}+E^{\prime} \wedge \Lambda=0 ;\left[E^{\prime}, \Lambda\right]+\left[E, \Lambda^{\prime}\right]=0$, and these are the conditions that assure the compatibility of the Jacobi structures $(\Lambda, E)$ and $\left(\Lambda^{\prime}, E^{\prime}\right)$ (see $\left.[20]\right)$.

\section{Null Dirac structures and characteristic pairs}

Let $\left((A, \phi),\left(A^{*}, W\right)\right)$ be a generalized Lie bialgebroid and $D \subset A$ a subbundle of $A$. We denote by $D^{\perp} \subset A^{*}$ the conormal bundle of $D$,

$$
D^{\perp}=\left\{\alpha \in A^{*}:\langle\alpha, X\rangle=0, \forall X \in D\right\} .
$$


Proposition 5.1. The subbundle $L=D \oplus D^{\perp}$ of $A \oplus A^{*}$ is a Dirac structure for the generalized Courant algebroid $\left(A \oplus A^{*}, \phi+W\right)$ if and only if $D$ and $D^{\perp}$ are Lie subalgebroids of $A$ and $A^{*}$, respectively. In this case, $L$ is said to be a null Dirac structure.

Proof: For any $X_{1}+\alpha_{1}, X_{2}+\alpha_{2} \in L=D \oplus D^{\perp}$,

$$
\left(X_{1}+\alpha_{1}, X_{2}+\alpha_{2}\right)_{ \pm}=\frac{1}{2}\left(\left\langle\alpha_{1}, X_{2}\right\rangle \pm\left\langle\alpha_{2}, X_{1}\right\rangle\right)=0
$$

and $L$ is a maximal isotropic subbundle of $A \oplus A^{*}$.

If $L$ is a Dirac structure for $\left(A \oplus A^{*}, \phi+W\right)$, then $L$ is integrable, i.e. $L$ is closed with respect to the bracket $\llbracket ., . \rrbracket$ given by $(23)$.

For any sections $X_{1}$ and $X_{2}$ of $D \subset L$, we compute

$$
\llbracket X_{1}+0, X_{2}+0 \rrbracket=\left[X_{1}, X_{2}\right]+0 \in \Gamma(L) .
$$

Therefore, $\left[X_{1}, X_{2}\right] \in \Gamma(D)$ and $D$ is a Lie subalgebroid of $A$ ([17]). An analogous reasoning shows that $D^{\perp}$ is a Lie subalgebroid of $A^{*}$.

Conversely, let us suppose that $D$ and $D^{\perp}$ are Lie subalgebroids of $A$ and $A^{*}$, respectively. Since we have, for any sections $X_{1}+\alpha_{1}, X_{2}+\alpha_{2}$ of $L$,

$$
\begin{aligned}
\llbracket X_{1}+\alpha_{1}, X_{2}+\alpha_{2} \rrbracket & =\left(\left[X_{1}, X_{2}\right]+\mathcal{L}_{* \alpha_{1}}^{W} X_{2}-\mathcal{L}_{* \alpha_{2}}^{W} X_{1}\right) \\
& +\left(\left[\alpha_{1}, \alpha_{2}\right]_{*}+\mathcal{L}_{X_{1}}^{\phi} \alpha_{2}-\mathcal{L}_{X_{2}}^{\phi} \alpha_{1}\right),
\end{aligned}
$$

for concluding that $L$ is integrable, we only have to verify that $\mathcal{L}_{* \alpha_{1}}^{W} X_{2}$ and $\mathcal{L}_{* \alpha_{2}}^{W} X_{1}$ (resp. $\mathcal{L}_{X_{1}}^{\phi} \alpha_{2}$ and $\left.\mathcal{L}_{X_{2}}^{\phi} \alpha_{1}\right)$ are sections of $D$ (resp. $D^{\perp}$ ). Or, if $\beta$ is a section of $D^{\perp}$,

$$
\begin{aligned}
\left\langle\beta, \mathcal{L}_{* \alpha_{1}}^{W} X_{2}\right\rangle & =\left\langle\beta, \mathcal{L}_{* \alpha_{1}} X_{2}\right\rangle+\left\langle\alpha_{1}, W\right\rangle \underbrace{\left\langle\beta, X_{2}\right\rangle}_{=0} \\
& =a_{*}^{\perp}\left(\alpha_{1}\right)\left(\left\langle\beta, X_{2}\right\rangle\right)-\left\langle\left[\beta, \alpha_{1}\right]_{*}, X_{2}\right\rangle \\
& =0,
\end{aligned}
$$

where $a_{*}^{\perp}$ stands for the anchor of the Lie algebroid $D^{\perp}$. Therefore, $\mathcal{L}_{* \alpha_{1}}^{W} X_{2} \in$ $\Gamma(D)$ and, in the same way, one has $\mathcal{L}_{* \alpha_{2}}^{W} X_{1} \in \Gamma(D)$. Similarly, one can show that $\mathcal{L}_{X_{1}}^{\phi} \alpha_{2}$ and $\mathcal{L}_{X_{2}}^{\phi} \alpha_{1}$ are sections of $D^{\perp}$. 
Let us now recall the notion of characteristic pair, introduced in [15], which provides Dirac structures generalizing both the case of a graph of a bivector field (treated in the previous section) and that of a null Dirac structure. See also [1] where the authors detailed some issues on characteristic pairs.

Let $A$ be a vector bundle, $D \subset A$ a subbundle of $A$ and $\Omega$ a bivector field, $\Omega \in \Gamma\left(\bigwedge^{2} A\right)$. Consider the subbundle $L$ of $A \oplus A^{*}$, given by

$$
L=\left\{X+\Omega^{\#} \alpha+\alpha, X \in D, \alpha \in D^{\perp}\right\}=D \oplus \operatorname{graph}\left(\left.\Omega^{\#}\right|_{D^{\perp}}\right) .
$$

Clearly $L \subset A \oplus A^{*}$ is maximally isotropic with respect to the symmetric bilinear form (21). In what follows, we will assume that $D=L \cap A$ is of constant rank.

Definition $5.2([15])$. The pair $(D, \Omega)$ is called the characteristic pair of the subbundle $L$ of $A \oplus A^{*}$ given by (63), while $D=L \cap A$ is called the characteristic subbundle of $L$.

As it is remarked in [15], the restricted bundle map $\left.\Omega^{\#}\right|_{D^{\perp}}$ is equivalent to a bivector field on the quotient bundle $A / D$. Thus, two characteristic pairs $\left(D_{1}, \Omega_{1}\right)$ and $\left(D_{2}, \Omega_{2}\right)$ determine the same subbundle $L$ defined by $(63)$ if and only if

$$
D_{1}=D_{2} \quad \text { and } \quad \Omega_{1}-\Omega_{2}=0(\bmod D),
$$

where by $\Omega_{1}-\Omega_{2}=0(\bmod D)$ we mean $\left(\Omega_{1}^{\#} \alpha-\Omega_{2}^{\#} \alpha\right) \in D, \forall \alpha \in D^{\perp}$.

We are interested in characteristic pairs for the case where $\left((A, \phi),\left(A^{*}, W\right)\right)$ is a generalized Lie bialgebroid.

Theorem 5.3. Let $\left((A, \phi),\left(A^{*}, W\right)\right)$ be a generalized Lie bialgebroid and $L \subset$ $A \oplus A^{*}$ a maximal isotropic subbundle of $A \oplus A^{*}$ defined by a characterisitc pair $(D, \Omega)$, i.e

$$
L=\left\{X+\Omega^{\#} \alpha+\alpha, X \in D, \alpha \in D^{\perp}\right\}=D \oplus \operatorname{graph}\left(\left.\Omega^{\#}\right|_{D^{\perp}}\right) .
$$

Then $L$ is a Dirac structure for the generalized Courant algebroid $(A \oplus$ $\left.A^{*}, \phi+W\right)$ if and only if:

i) $D$ is a Lie subalgebroid of $A$;

ii) $d_{*}^{W} \Omega+\frac{1}{2}[\Omega, \Omega]^{\phi}=0(\bmod D)$;

iii) for any $\alpha, \beta \in \Gamma\left(D^{\perp}\right),[\alpha, \beta]_{*}+[\alpha, \beta]_{\Omega} \in \Gamma\left(D^{\perp}\right)$, where $[., .]_{\Omega}$ is the bracket (51). 
Proof: We only have to verify that the closedness of $L$ is equivalent to conditions i), ii) and iii).

If $X+\Omega^{\#} \alpha+\alpha$ and $Y+\Omega^{\#} \beta+\beta$ are any sections of $L=D \oplus \operatorname{graph}\left(\left.\Omega^{\#}\right|_{D^{\perp}}\right)$, then

$$
\begin{aligned}
& \llbracket X+\Omega^{\#} \alpha+\alpha, Y+\Omega^{\#} \beta+\beta \rrbracket= \\
& \quad=\llbracket X, Y \rrbracket+\llbracket X, \Omega^{\#} \beta+\beta \rrbracket+\llbracket \Omega^{\#} \alpha+\alpha, Y \rrbracket+\llbracket \Omega^{\#} \alpha+\alpha, \Omega^{\#} \beta+\beta \rrbracket .(64)
\end{aligned}
$$

Concerning the first term of the second member of equation (64), $\llbracket X, Y \rrbracket=$ $[X, Y] \in \Gamma(D)$ if and only if $D$ is a Lie subalgebroid of $A$ (condition i)). The second and third terms of second member of (64) are of the same type.

Or,

$$
\begin{aligned}
\llbracket X, \Omega^{\#} \beta+\beta \rrbracket \stackrel{(23)}{=} & \left(\left[X, \Omega^{\#} \beta\right]-\mathcal{L}_{* \beta}^{W} X\right)+\mathcal{L}_{X}^{\phi} \beta \\
= & \left(\left[X, \Omega^{\#} \beta\right]-\mathcal{L}_{* \beta}^{W} X-\Omega^{\#}\left(\mathcal{L}_{X}^{\phi} \beta\right)\right)+\Omega^{\#}\left(\mathcal{L}_{X}^{\phi} \beta\right)+\mathcal{L}_{X}^{\phi} \beta .
\end{aligned}
$$

Moreover, for any $Z \in \Gamma(D)$,

$$
\begin{aligned}
\left\langle\mathcal{L}_{X}^{\phi} \beta, Z\right\rangle & =\left\langle\mathcal{L}_{X} \beta, Z\right\rangle+\langle\phi, X\rangle \underbrace{\langle\beta, Z\rangle}_{=0} \\
& =a(X)(\langle\beta, Z\rangle)-\langle\beta,[X, Z]\rangle \\
& =-\langle\beta,[X, Z]\rangle
\end{aligned}
$$

and so, $\mathcal{L}_{X}^{\phi} \beta \in \Gamma\left(D^{\perp}\right)$ if and only if $D$ is a Lie subalgebroid of $A$ (condition i)).

In this case, from (65) we deduce that

$\llbracket X, \Omega^{\#} \beta+\beta \rrbracket \in \Gamma(L)$ if and only if $\left[X, \Omega^{\#} \beta\right]-\mathcal{L}_{* \beta}^{W} X-\Omega^{\#}\left(\mathcal{L}_{X}^{\phi} \beta\right) \in \Gamma(D)$.

With $\alpha \in \Gamma\left(D^{\perp}\right)$, we compute 


$$
\begin{aligned}
&\left\langle\alpha,\left[X, \Omega^{\#} \beta\right]-\mathcal{L}_{* \beta}^{W} X-\Omega^{\#}\left(\mathcal{L}_{X}^{\phi} \beta\right)\right\rangle= \\
&=-\left\langle\alpha,\left[\Omega^{\#} \beta, X\right]\right\rangle-a_{*}(\beta)(\langle\alpha, X\rangle)+\left\langle[\beta, \alpha]_{*}, X\right\rangle+a(X)\left(\left\langle\beta, \Omega^{\#} \alpha\right\rangle\right) \\
&-\left\langle\beta,\left[X, \Omega^{\#} \alpha\right]\right\rangle+\langle\phi, X\rangle \Omega(\alpha, \beta) \\
&=\left\langle[\beta, \alpha]_{*}, X\right\rangle-\left\langle\alpha,\left[\Omega^{\#} \beta, X\right]\right\rangle-\left\langle\beta,\left[X, \Omega^{\#} \alpha\right]\right\rangle+\left\langle d^{\phi}(\Omega(\alpha, \beta)), X\right\rangle \\
&=\left\langle[\beta, \alpha]_{*}+\mathcal{L}_{\Omega^{\#} \beta} \alpha-\mathcal{L}_{\Omega^{\#} \alpha} \beta+d^{\phi}(\Omega(\alpha, \beta)), X\right\rangle \\
&=-\left\langle[\alpha, \beta]_{*}+[\alpha, \beta]_{\Omega}, X\right\rangle .
\end{aligned}
$$

So (66) is equivalent to

$$
\llbracket X, \Omega^{\#} \beta+\beta \rrbracket \in \Gamma(L) \text { if } \quad \text { and } \quad \text { only } \quad \text { if }[\alpha, \beta]_{*}+[\alpha, \beta]_{\Omega} \in \Gamma\left(D^{\perp}\right)
$$

(condition iii)).

For the last term of the second member of equation (64), a straightforward calculation, using (51), (53), (55) and (56), gives

$$
\begin{aligned}
\llbracket \Omega^{\#} \alpha+\alpha, \Omega^{\#} \beta+\beta \rrbracket= & \left(d_{*}^{W} \Omega+\frac{1}{2}[\Omega, \Omega]^{\phi}\right)(\alpha, \beta) \\
& +\Omega^{\#}\left([\alpha, \beta]_{*}+[\alpha, \beta]_{\Omega}\right)+\left([\alpha, \beta]_{*}+[\alpha, \beta]_{\Omega}\right) \cdot(69)
\end{aligned}
$$

So, we conclude that the bracket (64) is a section of $L$ if and only if the conditions i), ii) and iii) hold.

\section{Triangular generalized Lie bialgebroids and Dirac structures}

In this section we present a version of Theorem 5.3 for the case of a triangular generalized Lie bialgebroid. First, let us recall some results from [6].

Theorem $6.1([6])$. Let $(A,[.,], a$.$) be a Lie algebroid over M, \phi \in \Gamma\left(A^{*}\right)$ a 1-cocycle and $P \in \Gamma\left(\bigwedge^{2} A\right)$ a bivector field such that $[P, P]^{\phi}=0$. Then,

i) $\left(A^{*},[.,]_{P}, a \circ P^{\#}\right)$ is a Lie algebroid over $M$, where $[.,]_{P}$ is the bracket (51) associated with $P$;

ii) $W=-P^{\#}(\phi) \in \Gamma(A)$ is a 1-cocycle;

iii) the pair $\left((A, \phi),\left(A^{*}, W\right)\right)$ is a generalized Lie bialgebroid.

Definition $6.2([6])$. A generalized Lie bialgebroid $\left((A, \phi),\left(A^{*}, W\right)\right)$ is said to be a triangular generalized Lie bialgebroid if there exists $P \in \Gamma\left(\bigwedge^{2} A\right)$ 
such that $[P, P]^{\phi}=0$, the Lie bracket on $\Gamma\left(A^{*}\right)$ is $[., .]_{P}$, the anchor on $A^{*}$ is $\left(a \circ P^{\#}\right)$ and the 1 -cocycle $W$ is given by $W=-P^{\#}(\phi)$.

We will denote by $\left((A, \phi),\left(A^{*}, W\right), P\right)$ a triangular generalized Lie bialgebroid.

Example $6.3([6])$. Let $(M, \Lambda, E)$ be a Jacobi manifold. Then $((T M \times$ $\left.\mathbb{R},(0,1)),\left(T^{*} M \times \mathbb{R},(-E, 0)\right),(\Lambda, E)\right)$ is a triangular generalized Lie bialgebroid. In fact,

- $[(\Lambda, E),(\Lambda, E)]^{(0,1)}=0$, because $(M, \Lambda, E)$ is a Jacobi manifold;

- for all sections $(\alpha, f)$ and $(\beta, g)$ of $T^{*} M \times \mathbb{R}$,

$$
\begin{aligned}
{[(\alpha, f),(\beta, h)]_{(\Lambda, E)}=} & \mathcal{L}_{(\Lambda, E)^{\#}(\alpha, f)}^{(0,1)}(\beta, g)-\mathcal{L}_{(\Lambda, E)^{\#(\beta, g)}}^{(0,1)}(\alpha, f) \\
& -d^{(0,1)}((\Lambda, E)((\alpha, f),(\beta, g)))
\end{aligned}
$$

- the anchor is $\pi \circ(\Lambda, E)^{\#}$;

- $-(\Lambda, E)^{\#}(0,1) \stackrel{(2)}{=}(-E, 0)=W$.

Proposition 6.4. Let $\left((A, \phi),\left(A^{*}, W\right), P\right)$ be a triangular generalized Lie bialgebroid and $L \subset A \oplus A^{*}$ a maximal isotropic subbundle of $A \oplus A^{*}$ defined by a characteristic pair $(D, \Omega)$, i.e. $L=D \oplus \operatorname{graph}\left(\left.\Omega^{\#}\right|_{D^{\perp}}\right)$. Then $L$ is a Dirac structure for the generalized Courant algebroid $\left(A \oplus A^{*}, \phi+W\right)$ if and only if

1) $D$ is a Lie subalgebroid of $A$;

2) $[P+\Omega, P+\Omega]^{\phi}=0(\bmod D)$;

3) for any $Y \in \Gamma(D), \mathcal{L}_{Y}^{\phi}(P+\Omega)=0(\bmod D)$.

Proof: We will show that conditions 2) and 3) are equivalent to ii) and iii) of Theorem 5.3, respectively. For any bivector field $\Omega \in \Gamma\left(\bigwedge^{2} A\right)$, we have $d_{*}^{W} \Omega=[P, \Omega]^{\phi}$. Moreover,

$$
[P+\Omega, P+\Omega]^{\phi}=2[P, \Omega]^{\phi}+[\Omega, \Omega]^{\phi}
$$

and so we have

$$
\begin{aligned}
d_{*}^{W} \Omega+\frac{1}{2}[\Omega, \Omega]^{\phi} & =[P, \Omega]^{\phi}+\frac{1}{2}[\Omega, \Omega]^{\phi} \\
& =\frac{1}{2}[P+\Omega, P+\Omega]^{\phi},
\end{aligned}
$$

which proves the equivalence of 2) and ii) of Theorem 5.3. 
On the other hand, for any sections $\alpha$ and $\beta$ of $A^{*}$, it is immediate to verify that

$$
[\alpha, \beta]_{P+\Omega}=[\alpha, \beta]_{P}+[\alpha, \beta]_{\Omega}
$$

where $[., .]_{P+\Omega}$ and $[., .]_{\Omega}$ are the brackets (51) defined by the bivector fields $P+\Omega$ and $\Omega$, respectively, and $[., .]_{P}$, also given by (51), is the Lie bracket on $\Gamma\left(A^{*}\right)$.

If $Y$ is any section of $D$ then, from (67) we deduce

$$
\left\langle[\alpha, \beta]_{P}+[\alpha, \beta]_{\Omega}, Y\right\rangle=\left\langle\beta,\left[Y, \Omega^{\#} \alpha\right]-\mathcal{L}_{* \alpha}^{W} Y-\Omega^{\#}\left(\mathcal{L}_{Y}^{\phi} \alpha\right)\right\rangle,
$$

where $[., .]_{P}$ plays the role of $[.,]_{*}$. Taking into account that $\left\langle[\alpha, \beta]_{P}, Y\right\rangle=$ $-\left\langle\beta, \mathcal{L}_{* \alpha}^{W} Y\right\rangle$ we obtain, for any $\Omega \in \Gamma\left(\bigwedge^{2} A\right)$,

$$
\left\langle[\alpha, \beta]_{\Omega}, Y\right\rangle=\left\langle\beta,\left[Y, \Omega^{\#} \alpha\right]-\Omega^{\#}\left(\mathcal{L}_{Y}^{\phi} \alpha\right)\right\rangle .
$$

For $P+\Omega \in \Gamma\left(\bigwedge^{2} A\right),(73)$ turns to

$$
\left\langle[\alpha, \beta]_{P+\Omega}, Y\right\rangle=\left\langle\beta,\left[Y,(P+\Omega)^{\#} \alpha\right]-(P+\Omega)^{\#}\left(\mathcal{L}_{Y}^{\phi} \alpha\right)\right\rangle
$$

or, equivalently,

$$
\left\langle[\alpha, \beta]_{P+\Omega}, Y\right\rangle=\left\langle\beta,\left(\mathcal{L}_{Y}^{\phi}(P+\Omega)\right)^{\#} \alpha\right\rangle .
$$

Then we conclude that

$$
[\alpha, \beta]_{P+\Omega}=[\alpha, \beta]_{P}+[\alpha, \beta]_{\Omega} \in \Gamma\left(D^{\perp}\right) \Leftrightarrow \mathcal{L}_{Y}^{\phi}(P+\Omega)=0(\bmod D) .
$$

\section{Acknowledgments}

Both authors were partially supported by CMUC-FCT.

\section{References}

[1] J. Clemente-Gallardo and J. M. Nunes da Costa: Dirac-Nijenhuis structures, preprint.

2] T. J. Courant: Dirac manifolds, Trans. Amer. Math. Soc. 319, 2 (1990) 631-661.

[3] T. J. Courant and A. Weinstein: Beyond Poisson structures, Séminaire Sud-Rhodanien de géométrie, Travaux en Cours, vol 27, Hermann, Paris, 1988, pp 39-49.

[4] I Dorfman: Dirac structures and integrability of nonlinear evolution equations, Nonlinear Science: Theory and Applications, Wiley, Chichester, 1993. 
[5] J. Grabowski and G. Marmo: Jacobi structures revisited, J. Phys. A: Math.Gen. 34 (2001) 10975-10990.

[6] D. Iglesias and J. C. Marrero: Generalized Lie bialgebroids and Jacobi structures, J. Geom. Phys. 40 (2001) 176-200.

[7] D. Iglesias and J. C. Marrero: Lie algebroid foliations and $\mathcal{E}^{1}(M)$-Dirac structures, J. Phys. A: Math.Gen. 35 (2002) 4085-4104.

[8] D. Iglesias-Ponte and J. C. Marrero: Generalized Lie Bialgebroids and Strong Jacobi-Nijenhuis Structures, Extracta Mathematicae 17, 2 (2002) 259-271.

[9] Y. Kerbrat and Z. Souici-Benhammadi: Variétés de Jacobi et groupoïdes de contact, C. R. Acad. Sci. Paris, Série I, 317 (1993) 81-86.

[10] Y. Kosmann-Schwarzbach: Exact Gerstenhaber algebras and Lie bialgebroids, Acta Appl. Math. 41 (1995) 153-165.

[11] Y. Kosmann-Schwarzbach: The Lie Bialgebroid of a Poisson-Nijenhuis Manifold, Lett. in Math. Phys. 38 (1996) 421-428.

[12] Y. Kosmann-Schwarzbach and F. Magri: Poisson-Nijenhuis structures, Ann. Inst. Henri Poincaré A 53 (1990) 35-81.

[13] M. de León, J. C. Marrero and E. Padrón: H-Chevalley-Eilenberg cohomology of a Jacobi manifold and Jacobi-Chern class, C. R. Acad. Sci. Paris 325, Série I (1997) 405-410.

[14] A. Lichnerowicz: Les variétés de Jacobi et leurs algèbres de Lie associées, J. Math. pures et appl. 57 (1978) 453-488.

[15] Z. Liu: Some remarks on Dirac structures and Poisson reductions, in Poisson Geometry, Banach Center Publications, Vol. 51 (2000) 165-173.

[16] Z. Liu, A. Weinstein and P. Xu: Manin triples for Lie bialgebroids, J. Diff. Geom. 45 (1997) $547-574$.

[17] K. Mackenzie: Lie groupoids and Lie algebroids in differential geometry, Cambridge University Press, 1987.

[18] K. Mackenzie and P. Xu: Lie bialgebroids and Poisson groupoids, Duke Math. J. 73 (1994) $415-452$.

[19] J. C. Marrero, J. Monterde and E. Padrón: Jacobi-Nijenhuis manifolds and compatible Jacobi structures, C. R. Acad. Sci. Paris 329 Série I (1999) 797-802.

[20] J. M. Nunes da Costa: Compatible Jacobi manifolds: geometry and reduction, J. Phys. A: Math. Gen 31 (1998) 1025-1033.

[21] J. M. Nunes da Costa: A characterization of strict Jacobi-Nijenhuis manifolds through the theory of Lie algebroids, Rep. on Math. Phys. 50, 3 (2002) 339-347.

[22] K. Uchino: Remarks on the definition of a Courant algebroid, Lett. Math. Phys. 60, 2 (2002) $171-175$.

[23] A. Wade: Conformal Dirac structures, Lett. Math. Phys. 53 (2000) 331-348.

J. M. Nunes DA Costa

Departamento de Matemática, Universidade de Coimbra, Apartado 3008, 3001-454 CoimBra, Portugal,

E-mail address: jmcosta@mat.uc.pt

J. Clemente-Gallardo

Departamento de Matemática, Universidade de Coimbra, Apartado 3008, 3001-454 CoimBra, Portugal,

E-mail address: jesus@mat.uc.pt 